\title{
Natural reaction orbitals
}

\author{
Shuichi Ebisawa ${ }^{1}$, Masatoshi Hasebe ${ }^{1}$, Takuro Tsutsumi ${ }^{1}$, Takao Tsuneda ${ }^{2,3}$, \\ Tetsuya Taketsugu 2,4 *
}

Correspondence to: Tetsuya Taketsugu (E-mail: take@sci.hokudai.ac.jp)

1 Graduate School of Chemical Sciences and Engineering, Hokkaido University, Sapporo 060-0810, Japan

2 Department of Chemistry, Faculty of Science, Hokkaido University, Sapporo 060-0810, Japan

3 Graduate School of Science Technology and Innovation, Kobe University, Nada-ku, Kobe, Hyogo 657-8501, Japan

4 Institute for Chemical Reaction Design and Discovery (WPI-ICReDD), Hokkaido University, Sapporo 001-0021, Japan

\begin{abstract}
Natural reaction orbital (NRO) for analyzing chemical reactions from the viewpoint of the electronic theory is presented. The pair of the occupied and virtual NROs that drives chemical reaction are automatically extracted from the solutions of the coupled-perturbed self-consistent-field (CPSCF) equation for the perturbation of the nuclear displacement along the reaction path. Applying the NRObased reaction analysis method to the $\mathrm{H}_{2} \mathrm{CO}^{-}+\mathrm{H}_{3} \mathrm{CCl}$ reaction, it was found that the sum of squared singular values of the solutions of the CPSCF equation gives the maximum peak at the transition state structure and the shoulder of the potential energy curve, which clearly explains the process of electron transfer and bond formation in this reaction. The NRO-based reaction analysis method has been demonstrated to be universal and robust for describing sophisticated reaction mechanisms.
\end{abstract}




\section{INTRODUCTION}

Molecular orbitals increase in importance from both experimental and theoretical points of view. In experimental studies, the phase of molecular orbitals becomes observable in multidimensional electron spectroscopy and molecular orbital tomography. The multidimensional electron spectroscopy provides molecular orbital patterns by measuring the bound energies to remove an electron from a molecular orbital using various sources and simultaneously by evaluating a new physical quantity as a parameter. ${ }^{1-3}$ On the other hand, molecular orbital tomography has recently attracted attention as a versatile experimental method to draw molecular orbital images by performing computed tomography scans of molecules using a strong photon laser. ${ }^{4,5}$ These methods can observe not only HOMO but also a certain number of occupied and unoccupied valence orbitals. Therefore, it becomes realized experimentally to trace chemical reactions based on molecular orbitals. In theoretical studies, it becomes possible to quantitatively provide molecular orbital energies. Occupied and unoccupied orbital energies are established to be identical to the minus sign of the corresponding ionization potentials and electron affinities, respectively. ${ }^{6}$ It is proven that long-range corrected density functional theory (DFT) ${ }^{7}$ accurately reproduces valence orbital energies by considerably satisfying Sham-Schlüter theorem ${ }^{8}$ for orbital energies. ${ }^{9}$ These progress in experimental and theoretical molecular orbital studies enhance expectations on the chemical reaction analysis based on molecular orbitals.

Reaction orbital theory has been developed to perform reaction analyses based on molecular orbitals. The representative reaction orbital theory is frontier orbital (FO) theory, ${ }^{10,11}$ which forms the foundation for the Woodward-Hoffmann rule ${ }^{12-16}$ of pericyclic reactions and has led to the development of various reaction orbital theories such as conceptual $\mathrm{DFT}^{17}$ and reactive orbital energy theory. ${ }^{18,19}$ Molecular orbitals are usually obtained as the eigenfunctions of one-electron selfconsistent field (SCF) equations like the Hartree-Fock and Kohn-Sham equations. However, these 
conventional molecular orbitals, which we call "canonical molecular orbitals (CMOs)," have two difficulties in analyzing reaction mechanisms based on reaction orbital theories:

1. Virtual CMOs are usually too delocalized to be analyzed, especially in high-quality calculations using diffuse basis functions, though the orbital shapes and the interactions between occupied and virtual orbitals are essentially needed to explain the reactivity of molecular systems in reaction orbital theories.

2. The number of CMOs is too large to be clearly analyzed, even confined to valence CMOs of relatively small molecules, and therefore makes it difficult to look for the molecular orbitals activating reactions.

In order to overcome these difficulties, a sophisticated method is required to search for clear localized molecular orbitals activating target reactions.

Several orbital-based analyses have been developed for analyzing chemical bonds and chemical events: Natural bond orbital (NBO) $)^{20,21}$ analysis has been used to interpret chemical bond structure in electronic wavefunction for a given geometrical structure. Intrinsic soft molecular orbital (ISMO) approach ${ }^{22}$ was proposed to specify important molecular orbitals for the response of electrons to distortions and external electrostatic fields, in which CMOs are transformed by the singular value decomposition (SVD) of the U matrix, which is obtained as solutions to the coupled-perturbed SCF (CPSCF) equation. ${ }^{23-27}$ Natural transition orbital (NTO) ${ }^{28}$ analysis is a technique for extracting occupied-virtual orbital pairs that characterize the excited state using the SVD of the one-electron transition density matrix. The recently proposed principal interacting orbital (PIO) approach ${ }^{29-31}$ also uses the SVD technique to analyze the density matrix in terms of interacting fragments in the molecule system. Though these orbital-based analyses are very useful for exploring chemical bonds and chemical events from the viewpoint of electronic wavefunctions, there has been no orbital-based reaction analysis methods explicitly considering the nuclear displacements in chemical reactions. 
In this study, we propose an orbital-based reaction analysis method generating occupiedvirtual orbital pairs that characterize the electronic wavefunction variation along the reaction pathway, by applying the SVD to the U matrix of the CPSCF equation. The generated occupied-virtual orbital pair is called a "natural reaction orbital (NRO)". This method is applied to the electron transfer reaction of formaldehyde anion $\left(\mathrm{H}_{2} \mathrm{CO}^{-}\right)$and methyl chloride $\left(\mathrm{CH}_{3} \mathrm{Cl}\right)$, which is known to have a posttransition-state bifurcation. ${ }^{32-36}$ After briefly explaining the CPSCF equation, the method for generating NROs is presented in Sec. II. In Sec. III, the NRO method is applied to the $\mathrm{H}_{2} \mathrm{CO}^{-}+\mathrm{CH}_{3} \mathrm{Cl}$ reaction, which is fully clarified for the electron transfer nature. 


\section{METHOD FOR GENERATING NATURAL REACTION ORBITALS}

In this section, we describe the procedure for generating natural reaction orbitals (NROs) based on the $\mathrm{U}$ matrix, which is the solution to the CPSCF equation. Henceforth, the subscripts $i\left(i=1, \ldots, N_{\mathrm{occ}}\right)$, $a\left(a=N_{\mathrm{occ}}+1, \ldots, N_{\mathrm{occ}}+N_{\mathrm{vir}}\right)$, and $p\left(p=1, \ldots, N_{\mathrm{occ}}+N_{\mathrm{vir}}\right)$ are used for occupied, virtual, and all spin orbitals, respectively, and the subscripts $\mu$ and $v$ are used for atomic orbitals (AO).

First, the CPSCF equation ${ }^{23-27}$ is briefly reviewed. The Hartree-Fock-Roothaan (HFR) equation is represented as

$$
\mathbf{F}(0) \mathbf{C}(0)=\mathbf{S}(0) \mathbf{C}(0) \mathbf{E}(0)
$$

where $\mathbf{F}, \mathbf{C}, \mathbf{S}$, and $\mathbf{E}$ are the Fock matrix, the molecular orbital coefficient matrix, the overlap matrix, and the diagonal orbital energy matrix, respectively, and 0 denotes the unperturbed nuclear coordinates. For a perturbed geometric structure shifted by an infinitesimal displacement parameter $\tau(>0)$ in the direction of the unit vector $\boldsymbol{n}$, the HFR equation is given by

$$
\mathbf{F}(\tau) \mathbf{C}(\tau)=\mathbf{S}(\tau) \mathbf{C}(\tau) \mathbf{E}(\tau)
$$

By expanding the respective matrices in Eq. (2) by powers of $\tau$ as

$$
\begin{aligned}
& \mathbf{F}(\tau)=\mathbf{F}(0)+\tau \mathbf{F}^{(1)}(0)+O\left(\tau^{2}\right) \\
& \mathbf{C}(\tau)=\mathbf{C}(0)+\tau \mathbf{C}^{(1)}(0)+O\left(\tau^{2}\right) \\
& \mathbf{S}(\tau)=\mathbf{S}(0)+\tau \mathbf{S}^{(1)}(0)+O\left(\tau^{2}\right) \\
& \mathbf{E}(\tau)=\mathbf{E}(0)+\tau \mathbf{E}^{(1)}(0)+O\left(\tau^{2}\right)
\end{aligned}
$$

and equating the first-order terms, we get

$$
\mathbf{F}^{(1)}(0) \mathbf{C}(0)+\mathbf{F}(0) \mathbf{C}^{(1)}(0)=\mathbf{S}^{(1)}(0) \mathbf{C}(0) \mathbf{E}(0)+\mathbf{S}(0) \mathbf{C}^{(1)}(0) \mathbf{E}(0)+\mathbf{S}(0) \mathbf{C}(0) \mathbf{E}^{(1)}(0) .
$$

Note that these first-order responses depend on the direction of $\boldsymbol{n}$. Henceforth, since Eq. (4) is given in the $\tau$-free form, we omit the 0 representing the nuclear coordinates. Multiplying both sides of Eq. (4) by $\mathbf{C}^{\dagger}$, the Hermitian conjugate of $\mathbf{C}$ from the left, and using Eq. (1), we obtain the following equation as

$$
\mathbf{C}^{\dagger} \mathbf{F}^{(1)} \mathbf{C}+\mathbf{E C}^{\dagger} \mathbf{S C} \mathbf{C}^{(1)}=\mathbf{C}^{\dagger} \mathbf{S}^{(1)} \mathbf{C E}+\mathbf{C}^{\dagger} \mathbf{S} \mathbf{C}^{(1)} \mathbf{E}+\mathbf{E}^{(1)}
$$


Introducing the matrices of $\tilde{\mathbf{F}}^{(1)}, \mathbf{U}^{(1)}$ and $\tilde{\mathbf{S}}^{(1)}$ in the form of

$$
\begin{aligned}
\tilde{\mathbf{F}}^{(1)} & =\mathbf{C}^{\dagger} \mathbf{F}^{(1)} \mathbf{C}, \\
\mathbf{U}^{(1)} & =\mathbf{C}^{\dagger} \mathbf{S} \mathbf{C}^{(1)}, \\
\tilde{\mathbf{S}}^{(1)} & =\mathbf{C}^{\dagger} \mathbf{S}^{(1)} \mathbf{C},
\end{aligned}
$$

Eq. (5) is rewritten as

$$
\mathbf{E} \mathbf{U}^{(1)}-\mathbf{U}^{(1)} \mathbf{E}=\widetilde{\mathbf{S}}^{(1)} \mathbf{E}-\tilde{\mathbf{F}}^{(1)}+\mathbf{E}^{(1)} .
$$

The first-order orthonormality condition for molecular orbitals is expressed as

$$
\mathbf{U}^{(1) \dagger}+\widetilde{\mathbf{S}}^{(1)}+\mathbf{U}^{(1)}=\mathbf{0}
$$

Solving Eqs. (7) and (8) yields $\mathbf{E}^{(1)}$ and the virtual-occupied block of $\mathbf{U}^{(1)}$. The equation to obtain the virtual-occupied block of $\mathbf{U}^{(1)}$ as

$$
\left(\epsilon_{a}-\epsilon_{i}\right) \mathrm{U}_{a i}^{(1)}=\epsilon_{i} \widetilde{\mathrm{S}}_{a i}^{(1)}-\widetilde{\mathrm{F}}_{a i}^{(1)}
$$

is called coupled perturbed Hartree-Fock (CPHF) equation where $\epsilon_{i}$ and $\epsilon_{a}$ are occupied and virtual orbital energies, respectively. The coupled perturbed Kohn-Sham (CPKS) equation is obtained by replacing the Fock matrix with an effective Hamiltonian matrix for the Kohn-Sham equation. For the details of the CPHF and CPKS equations, see Refs. 23-27.

Let us introduce the operator transforming the unperturbed occupied CMOs to the perturbed ones,

$$
\mathcal{T}(\tau, 0)=\sum_{p=1}^{N_{\mathrm{occ}}+N_{\mathrm{vir}}} n_{p}\left|\psi_{p}(\tau)\right\rangle\left\langle\psi_{p}(0)\left|=\sum_{i=1}^{N_{\mathrm{occ}}} \sum_{\mu, v} c_{\mu i}(\tau) c_{\nu i}^{*}(0)\right| \chi_{\mu}(\tau)\right\rangle\left\langle\chi_{\nu}(0)\right|,
$$

where $n_{p}$ is the occupation number of the $p$-th spin orbital (i.e. $n_{i}=1$ and $\left.n_{a}=0\right), \psi_{p}$ and $\chi_{\mu}$ are the $p$-th $\mathrm{CMO}$ and $\mu$-th $\mathrm{AO}$, respectively, and $\tau$ is the infinitesimal nuclear displacement parameter. Expanding $c_{\mu i}(\tau)$ by powers of $\tau$ gives the zeroth- and first-order transformation operators,

$$
\mathcal{T}^{(0)}(\tau, 0)=\sum_{i=1}^{N_{\text {occ }}} \sum_{\mu, v} c_{\mu i}(0) c_{v i}^{*}(0)\left|\chi_{\mu}(\tau)\right\rangle\left\langle\chi_{v}(0)\right|,
$$




$$
\mathcal{T}^{(1)}(\tau, 0)=\sum_{p=1}^{N_{\mathrm{occ}}+N_{\mathrm{vir}}} \sum_{i=1}^{N_{\mathrm{occ}}} \sum_{\mu, \nu} c_{\mu p}(0) \mathrm{U}_{p i}^{(1)}(0) c_{\nu i}^{*}(0)\left|\chi_{\mu}(\tau)\right\rangle\left\langle\chi_{v}(0)\right|
$$

where we used the definition of $\mathbf{U}^{(1)}$ as

$$
\mathbf{C}^{(1)}(0)=\mathbf{C}(0) \mathbf{U}^{(1)}(0) \text {. }
$$

The zeroth-order operator works only to translate the AOs without mixing the CMOs. On the other hand, the first-order operator causes mixing of CMOs as the AOs are translated. Actually, the translation of AOs and the mixing of CMOs can be separated as follows

$$
\mathcal{T}^{(1)}(\tau, 0)=\mathcal{T}^{(0)}(\tau, 0) \mathcal{U}^{(1)}(0) \text {, (14) }
$$

where the first-order mixing operator $U^{(1)}(0)$ is defined as

$$
\begin{array}{r}
u^{(1)}(0)=\sum_{p=1}^{N_{\text {occ }}+N_{\text {vir }}} \sum_{i=1}^{N_{\text {occ }}} \sum_{\mu, \nu} c_{\mu p}(0) \mathrm{U}_{p i}^{(1)}(0) c_{v i}^{*}(0)\left|\chi_{\mu}(0)\right\rangle\left\langle\chi_{v}(0)\right| \\
=\sum_{p=1}^{N_{\text {occ }}+N_{\text {vir }}} \sum_{i=1}^{N_{\text {occ }}} \mathrm{U}_{p i}^{(1)}(0)\left|\psi_{p}(0)\right\rangle\left\langle\psi_{i}(0)\right| .
\end{array}
$$

The occupied-occupied terms are a rather trivial part of the mixing because the Slater determinant is invariant under the first-order mixing between occupied orbitals. Accordingly, we define the firstorder one-electron transition density operator as

$$
\mathcal{U}_{\mathrm{vO}}^{(1)}(0)=\sum_{a=N_{\mathrm{occ}}+1}^{N_{\mathrm{occ}}+N_{\mathrm{vir}}} \sum_{i=1}^{N_{\mathrm{occ}}} \mathrm{U}_{a i}^{(1)}(0)\left|\psi_{a}(0)\right\rangle\left\langle\psi_{i}(0)\right|
$$

$u_{\mathrm{vO}}^{(1)}(0)$ is expanded with the basis functions in the unperturbed geometry. Consequently, the mixing between CMOs along the nuclear displacement can be analyzed in terms of the basis function in the unperturbed geometry. The singular value decomposition (SVD) of $\mathbf{U}_{\mathrm{VO}}^{(1)}(0)$ yields the $N_{\text {occ }} \times N_{\text {occ }}$ unitary matrix $\mathbf{O}$ and the $N_{\text {vir }} \times N_{\text {vir }}$ unitary matrix $\mathbf{V}$ as

$$
\mathbf{U}_{\mathrm{VO}}^{(1)}(0)=\mathbf{V} \boldsymbol{\Lambda} \mathbf{0}^{\dagger},
$$

where $\Lambda$ is a rectangular diagonal matrix of size $N_{\text {vir }} \times N_{\text {occ }}$. The non-negative real numbers in the 
diagonal part of $\boldsymbol{\Lambda}, \lambda_{i}\left(i=1, \ldots, N_{\text {occ }}\right)$, are called the singular values of $\mathbf{U}^{(1)}(0)$. The occupied and virtual CMOs are transformed by the unitary matrix $\mathbf{O}$ and the unitary matrix $\mathbf{V}$, respectively, as follows:

$$
\begin{gathered}
\left(\phi_{1}, \phi_{2}, \ldots, \phi_{N_{\mathrm{occ}}}\right)=\left(\psi_{1}, \psi_{2}, \ldots, \psi_{N_{\mathrm{occ}}}\right) \mathbf{0} \\
\left(\phi_{1}^{\prime}, \phi_{2}^{\prime}, \ldots, \phi_{N_{\mathrm{vir}}}^{\prime}\right)=\left(\psi_{N_{\mathrm{occ}}+1}, \psi_{N_{\mathrm{occ}}+2}, \ldots, \psi_{N_{\mathrm{occ}}+N_{\mathrm{vir}}}\right) \mathbf{V}
\end{gathered}
$$

The set of orbitals in Eqs. (18) and (19), i.e., $\left(\phi_{1}, \phi_{2}, \ldots, \phi_{N_{\text {occ }}}\right)$ and $\left(\phi_{1}^{\prime}, \phi_{2}^{\prime}, \ldots, \phi_{N_{\text {vir }}}^{\prime}\right)$, are referred to as natural reaction orbitals (NROs). Henceforth, virtual NROs will be marked with the prime symbol to avoid any misunderstanding. Singular values, $\lambda_{i}$, are assigned to $N_{\text {occ }}$ pairs of occupied NROs and virtual NROs: $\phi_{i}$ and $\phi_{i}^{\prime}$ (where we assume $N_{\text {occ }} \leq N_{\text {vir }}$ ). All the singular values for the rest of the virtual NROs are zero, i.e. $\lambda_{a}=0\left(a>N_{\text {occ }}\right)$.

Now consider the physical meaning of the singular value for the pair of occupied and virtual NROs. As shown in Eq. (14), the first-order orbital responses can be divided into mixing of orbitals in the unperturbed structure and subsequent translation of the AOs. The virtual-occupied orbital mixing changes the $i$-th $\mathrm{NRO}$ as

$$
\phi_{i}(0) \rightarrow \phi_{i}(0)+\tau \lambda_{i} \phi_{i}^{\prime}(0) \quad\left(i=1, \ldots, N_{\text {occ }}\right),
$$

where $\tau$ is the infinitesimal displacement parameter. This relation indicates that the virtual NRO mixes with the occupied NRO under the perturbation of nuclear coordinate displacement, and the corresponding singular value evaluates its mixing rate. Therefore, the occupied-virtual NRO pairs with large singular values are more correlated with nuclear coordinate displacement than those with small singular values.

There are two important properties of NROs. First, the relative phases of NRO pairs with nonzero singular values are uniquely determined for a given nuclear displacement direction. If we reverse the sign of the $i$-th column (i-th occupied NRO coefficient) of $\mathbf{0}^{\dagger}$ in Eq. (17), we must also reverse the sign of the $i$-th row ( $i$-th virtual NRO coefficient) of $\mathbf{V}$ in order for the equation to hold. Second, 
when the direction of nuclear displacement is reversed, the relative phase of each NRO pair is also reversed. This comes from the fact that the sign of $\mathbf{U}_{\mathrm{vO}}^{(1)}(0)$ is reversed when the direction of nuclear displacement is reversed. In the next section, we will discuss these two points again when applying NRO analysis to a specific chemical reaction.

As for the computational cost, the bottleneck for determining NROs is the step of solving the CPSCF equation to obtain the $\mathrm{U}$ matrix for a given structure. ${ }^{37}$ In the commonly used quantum chemical calculation program packages, the CPSCF equation is solved to calculate the analytic Hessian matrix, so the U matrix data can be obtained by performing normal mode analysis. However, $\mathrm{NRO}$ analysis does not require the $\mathrm{U}$ matrix for displacements in the direction of all degrees of freedom of the nucleus, but only the $U$ matrix for displacements in the reaction coordinate direction. If the CPSCF equation cannot be solved analytically, the U matrix can be obtained by numerical differentiation like $\mathbf{U}^{(1)} \sim(2 \tau)^{-1} \mathbf{C}^{\dagger}(0) \mathbf{S}(0)(\mathbf{C}(\tau)-\mathbf{C}(-\tau))$.

Finally, we would like to comment on the difference between the NRO method and the ISMO method. ${ }^{22}$ The ISMO method also transforms the CMO by applying SVD to the virtual-occupied term of $\mathbf{U}^{(1)}$ obtained from the CPSCF equation, but the perturbation is not a displacement of the nuclear coordinates but a fluctuation of the partial charges. In the viewpoint of solvent effects, ISMOs with large singular values are particularly important for the response of electrons to surrounding solvent molecules. On the other hand, NRO is concerned with electron transfer and bond rearrangement in chemical reaction processes, as seen in organic electron theory. In other words, NRO clarifies the mechanism of electron transfer from occupied orbital space to virtual orbital space when the structure changes along the reaction path, which is useful for understanding the reaction mechanism based on the orbital picture. 


\section{RESULTS AND DISCUSSION}

Applying the NROs to the electron transfer reaction between the formaldehyde anion and methyl chloride, we demonstrate how the NROs can represent the nature of electron transfers in the reaction. As shown in Fig. 1, this reaction is known to exhibit a post-transition-state bifurcation: the reactant cluster $\left(\mathrm{C}_{\mathrm{R}}\right)$ is transformed into either the $\mathrm{C}$-alkylation cluster $\left(\mathrm{C}_{\mathrm{SUB}(\mathrm{C})}\right)$ or the dissociative electron transfer cluster $\left(\mathrm{C}_{\mathrm{ET}}\right)$ through the common electron transfer transition state (ET-TS). ${ }^{32-36}$ For this reaction, the features of electron transfer have been investigated using a valence bond configuration mixing (VBCM) analysis. ${ }^{32-35}$ This reaction is, therefore, suitable to test the performance of the NRO analysis. We, therefore, apply the NROs to the intrinsic reaction coordinate (IRC) ${ }^{38}$ from $C_{R}$ to $C_{S U B(C)}$ through the ET-TS with keeping the $C_{\mathrm{s}}$ point group symmetry. The ET-TS structure optimization and the IRC and the $\mathrm{U}_{a i}^{(1)}$ calculations were carried out for the doublet ground state using the spinunrestricted Hartree-Fock (UHF) with the $6-31+\mathrm{G}(\mathrm{d}, \mathrm{p})$ basis set. ${ }^{39-42}$ All the calculations were performed using Gaussian16 Rev.C. $01 .{ }^{43}$

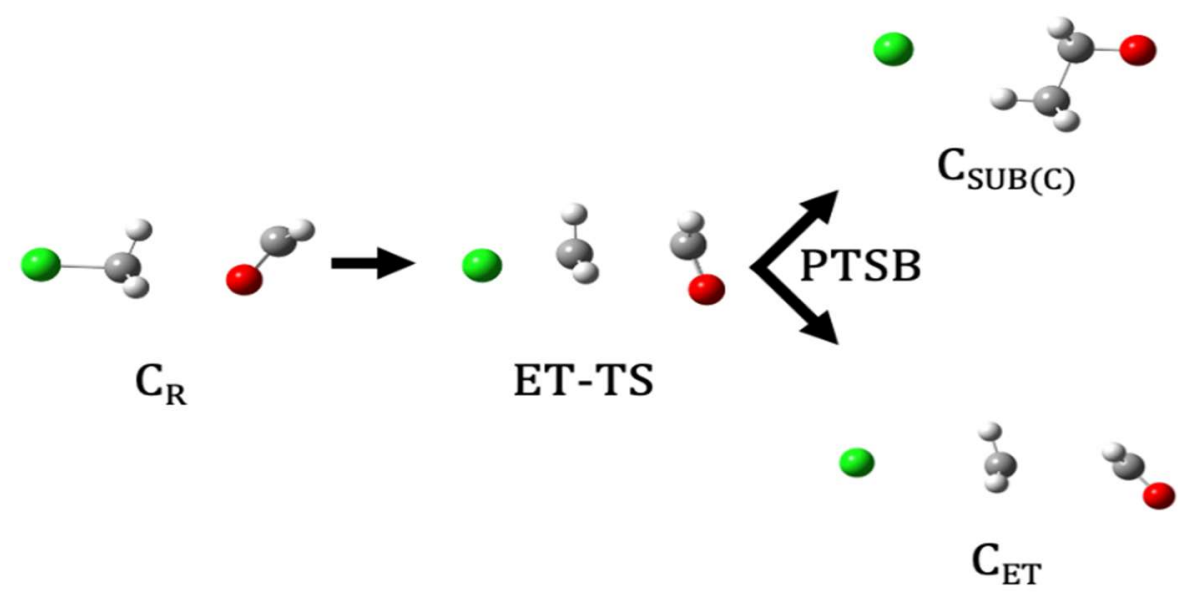

Figure 1. A schematic picture of the post-transition-state bifurcation (PTSB) in the electron transfer reaction of formaldehyde anion $\left(\mathrm{H}_{2} \mathrm{CO}^{-}\right)$and methyl chloride $\left(\mathrm{CH}_{3} \mathrm{Cl}\right)$ : the reactant cluster $\left(\mathrm{C}_{\mathrm{R}}\right)$ changes to the $\mathrm{C}$-alkylation cluster $\mathrm{C}_{\mathrm{SUB}(\mathrm{C})}$ or the dissociative electron transfer cluster $\mathrm{C}_{\mathrm{ET}}$ through the electron transfer transition state (ET-TS). The IRC from ET-TS to the product side leads to $\mathrm{C}_{\mathrm{SUB}(\mathrm{C}) \text {. }}$ 
The CMOs (from HOMO-4 to LUMO+14) of $\alpha$ - and $\beta$-spin electrons in ET-TS are shown in Fig. 2. Due to the doublet electronic state, the HOMO of the $\beta$-spin corresponds to the HOMO-1 of the $\alpha$-spin. This figure shows that the electronic distribution of the virtual CMOs tends to be too delocalized to easily discuss the electron transfers between the occupied CMOs and the virtual CMOs.

(a)
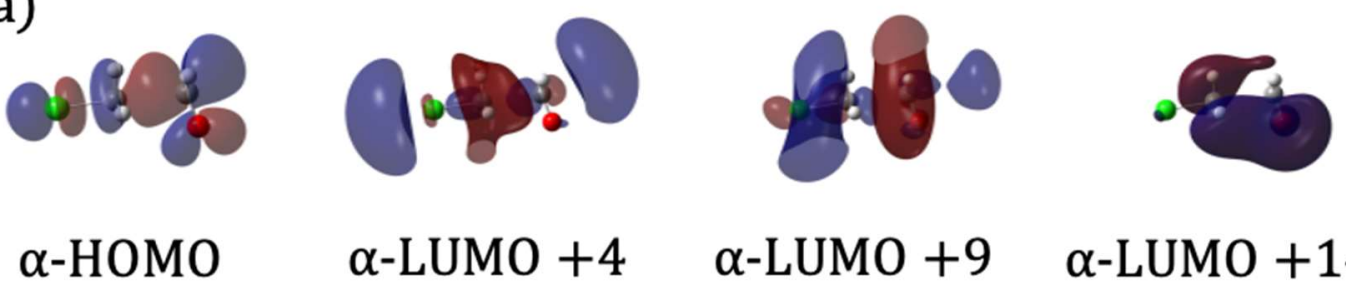

$\alpha$-LUMO +14
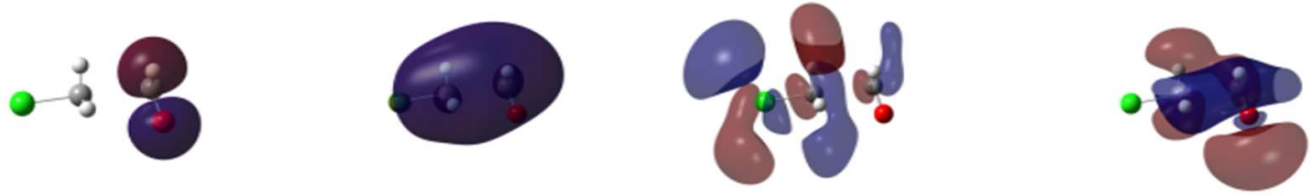

$$
\alpha \text {-HOMO -1 }
$$$$
\alpha \text {-LUMO +3 }
$$

$\alpha$-LUMO +8

$\alpha$-LUMO +13
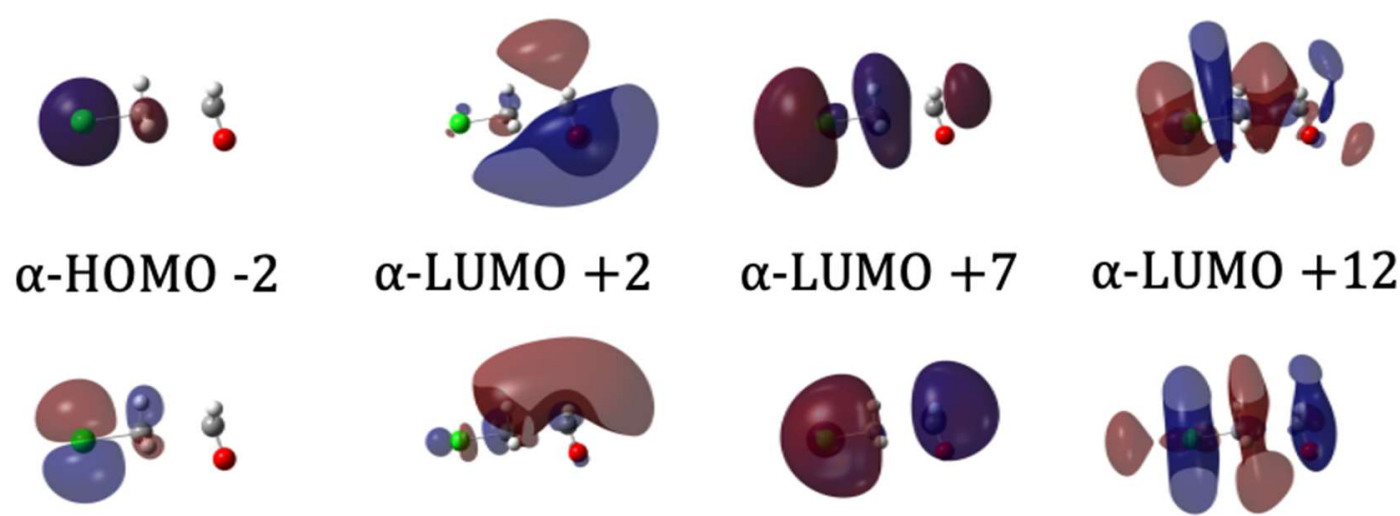

$\alpha$-LUMO +12
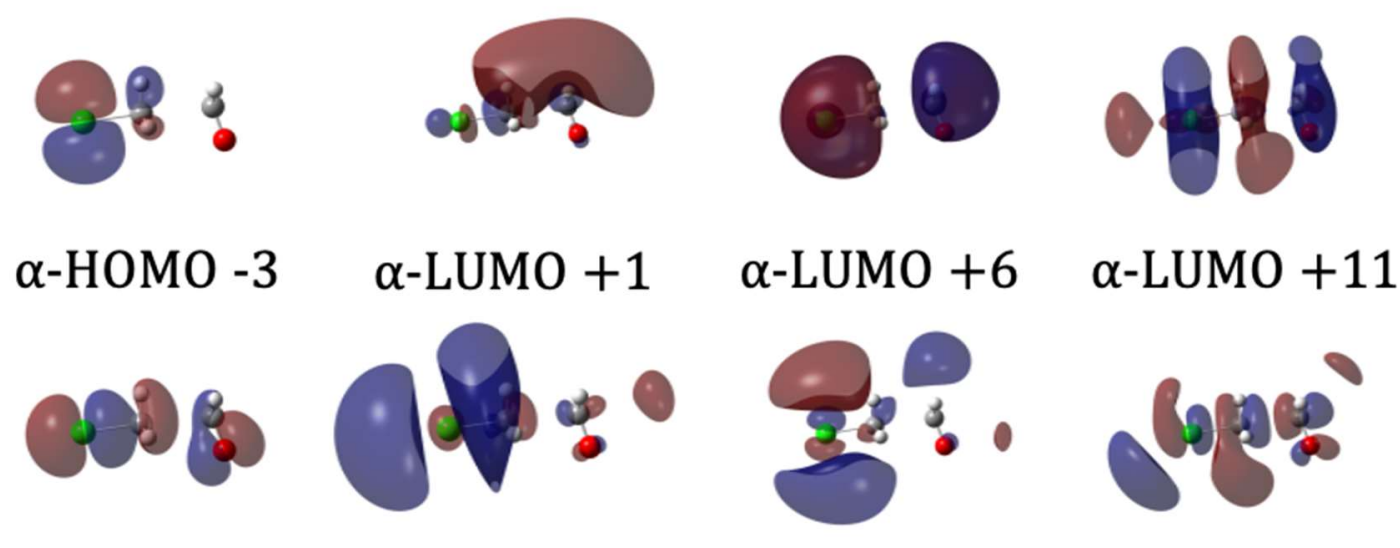

$\alpha$-HOMO -4

$\alpha$-LUMO
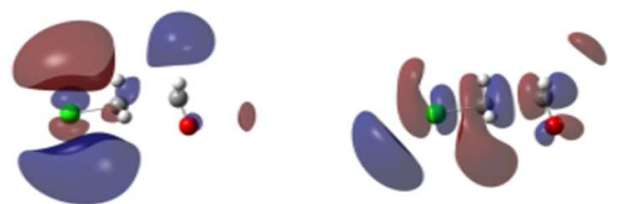

$\alpha$-LUMO +5

$\alpha$-LUMO +10 
(b)

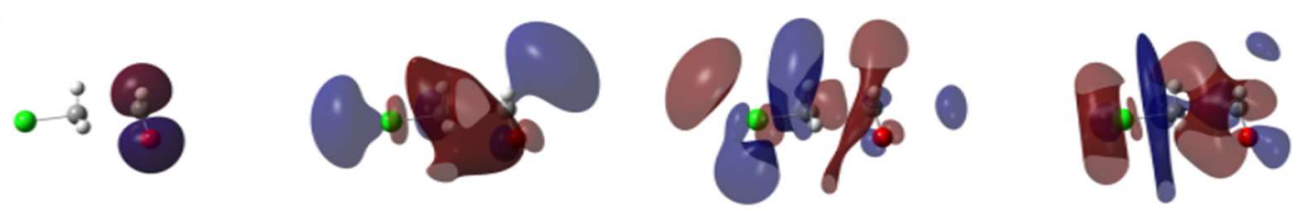

$\beta$-HOMO $\beta$-LUMO +4 $\beta$-LUMO +9 $\beta$-LUMO +14
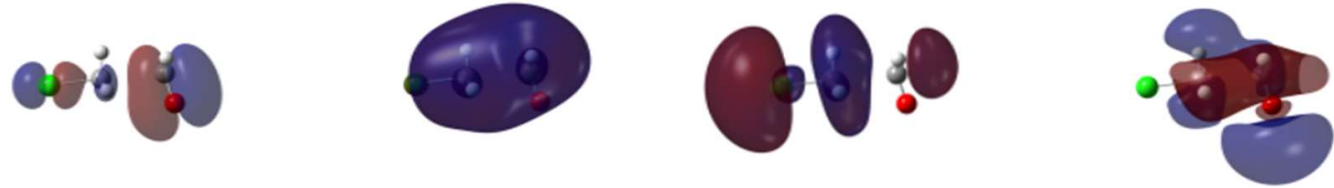

$\beta$-HOMO -1 $\beta$-LUMO +3 $\beta$-LUMO +8 $\beta$-LUMO +13

○०:
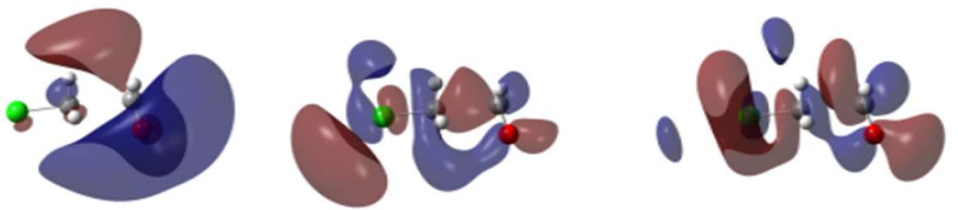

$\beta$-HOMO -2

$\beta$-LUMO +2

$\beta$-LUMO +7

$\beta$-LUMO +12
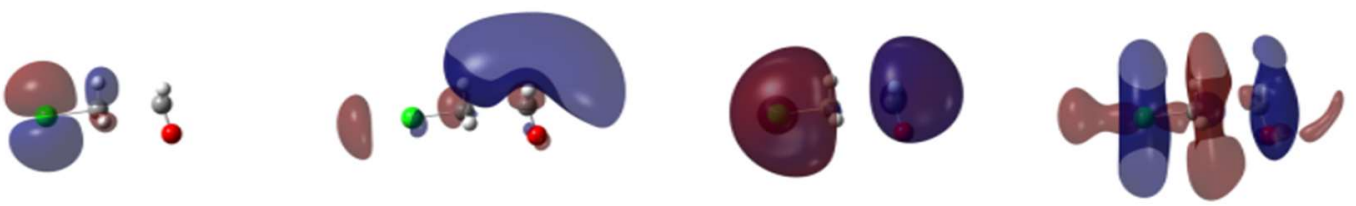

$\beta$-HOMO -3

$\beta$-LUMO +1

$\beta$-LUMO +6

$\beta$-LUMO +11
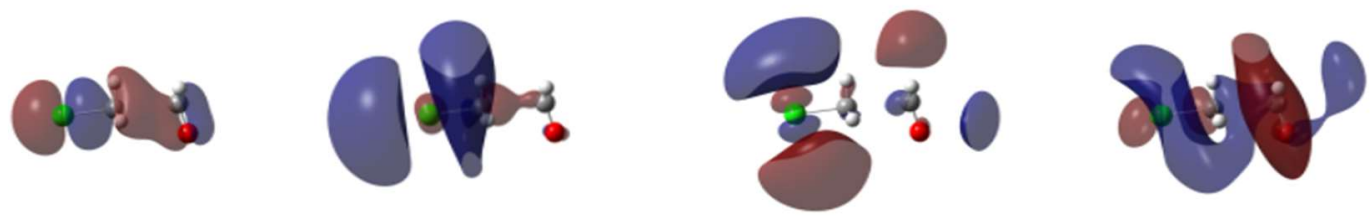

$\beta$-HOMO -4

$\beta$-LUMO

$\beta$-LUMO +5

$\beta$-LUMO +10

Figure 2. Five occupied and 15 virtual canonical molecular orbitals at the electron transfer transition state for (a) $\alpha$ - and (b) $\beta$-spin electrons with the isovalue of 0.02 . 
Figure 3 shows the square of the singular values of the $\mathbf{U}_{\mathrm{VO}}^{(1)}$ matrix in Eq. (17) for the $\alpha$ spin and $\beta$-spin orbitals and the NRO pairs with the maximum singular values $\left(\phi_{1}^{\alpha}\right.$ and $\left.\phi_{1}^{\prime \alpha}\right)$ for the nuclear displacements along the imaginary frequency mode of the ET-TS structure in Fig. 4. The singular value is an indicator for the extent to which the virtual NROs are mixed with the occupied NROs following the displacement of nuclei along the IRC. The singular value for the pair of $\phi_{1}^{\alpha}$ and $\phi_{1}^{\prime \alpha}$ is $0.362 \mathrm{amu}^{-1 / 2} \mathrm{bohr}^{-1}$, which accounts for the $94.8 \%$ of the $\alpha$-spin occupied-virtual orbital mixing. This backs up the ability of the NRO pair to explain the electron transfer in this reaction. This result also suggests that the NRO analysis solves the problem of CMOs that virtual CMOs are usually highly delocalized. The sum of the squared singular values of the $\beta$-spin orbitals $\left(0.020 \mathrm{amu}^{-1} \mathrm{bohr}^{-}\right.$ $\left.{ }^{2}\right)$ is much smaller than that of the $\alpha$-orbitals $\left(0.138 \mathrm{amu}^{-1} \mathrm{bohr}^{-2}\right)$. Note that this NRO analysis hardly depends on the basis set used. Actually, we obtained almost the same NROs in shape using both the $6-31+G(d, p)$ basis set and aug-cc-pVTZ basis set ${ }^{44-46}$ for this reaction (see APPENDIX), and also found that the contributions of major NROs are also hardly affected from the difference in the basis sets. This robustness of the NROs supports the reliability of the NRO-based analyses. 


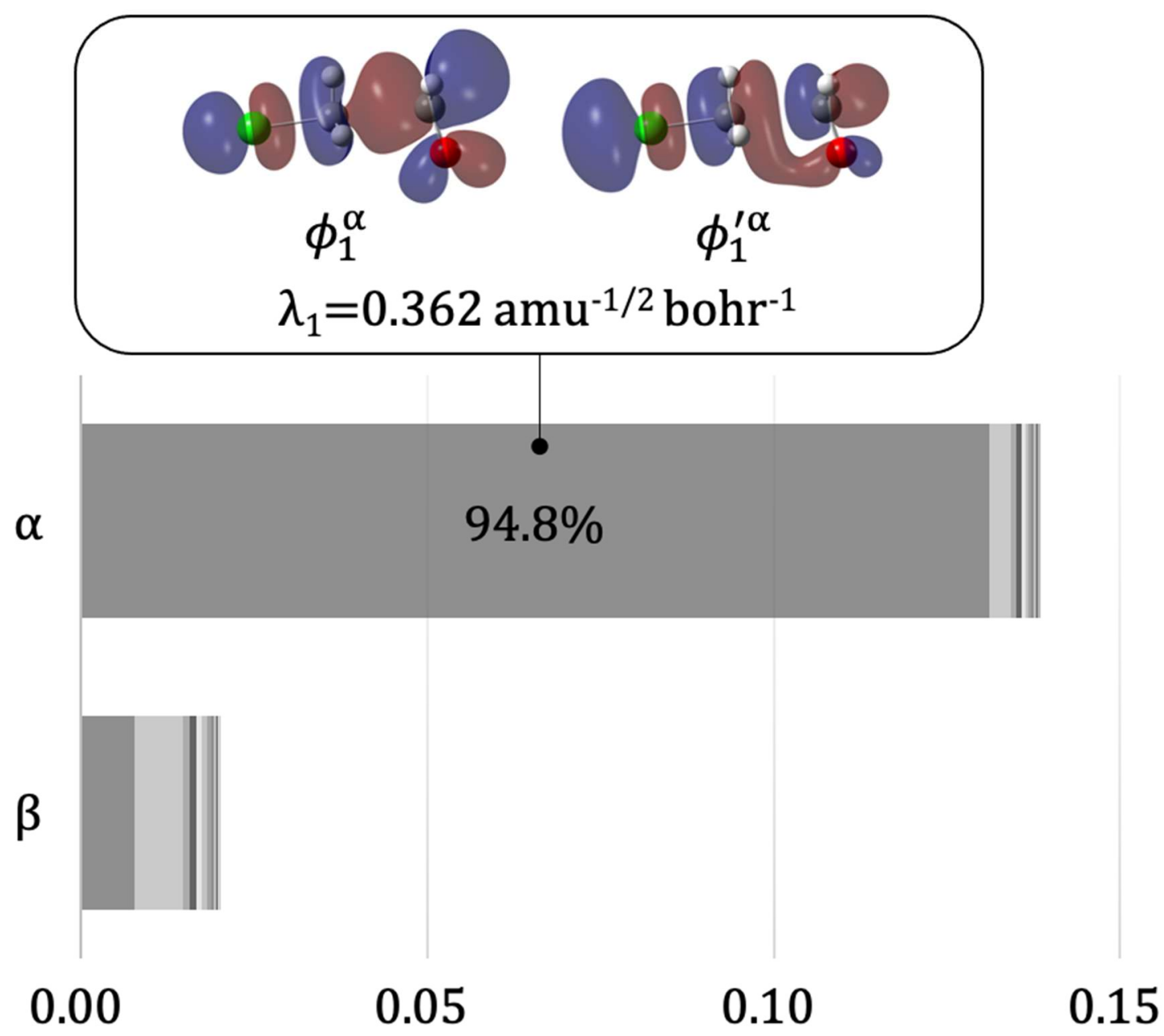

Square of singular values $\left[\mathrm{amu}^{-1} \mathrm{bohr}^{-2}\right]$

Figure 3. Distribution of the squared singular values of $\mathbf{U}_{\mathrm{VO}}^{(1)}$ for $\alpha$ - and $\beta$-spin NRO orbitals associated with the nuclear displacements along the imaginary frequency mode at the ET-TS structure of the $\mathrm{H}_{2} \mathrm{CO}^{-}+\mathrm{CH}_{3} \mathrm{Cl}$ reaction. The corresponding major $\mathrm{NRO}$ pair of the maximum singular value is also shown. 

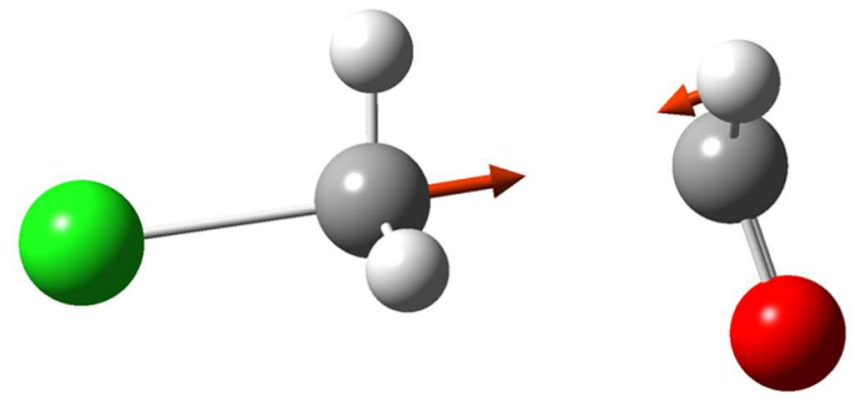

Figure 4. Normal mode vector of the imaginary frequency at the ET-TS structure of $\mathrm{H}_{2} \mathrm{CO}^{-}+\mathrm{CH}_{3} \mathrm{Cl}$ reaction (indicated by orange arrows). This corresponds to the nuclear displacement along the IRC producing the $\mathrm{C}_{\mathrm{SUB}(\mathrm{C})}$ structure in Fig. 1.

As shown in Fig. 3, the occupied NRO, $\phi_{1}^{\alpha}$, transforms as $\phi_{1}^{\alpha}+0.362 \tau \phi_{1}^{\prime \alpha}$ due to the virtual-occupied mixing caused by the nuclear displacement along the direction toward the product. As a result of this transformation, the electron density of formaldehyde decreases by mixing $\phi_{1}^{\alpha}$ and $\phi_{1}^{\prime \alpha}$ in the opposite-sign phases, while the electron density of methyl chloride increases by mixing $\phi_{1}^{\alpha}$ and $\phi_{1}^{\prime \alpha}$ in the same-sign phases (see Fig. 5). These results reveal the electron transfer from the formaldehyde anion to methyl chloride. For the opposite direction toward the reactants, the opposite process makes progress: $\phi_{1}^{\alpha} \rightarrow \phi_{1}^{\alpha}-0.362 \tau \phi_{1}^{\prime \alpha}$. As discussed in Sec. II, for the reversed direction of nuclear displacement, the relative phase of each NRO pair is reversed to give the minus sign of $\phi_{1}^{\prime \alpha}$ and the electron transfer from methyl chloride to formaldehyde anion proceeds. 

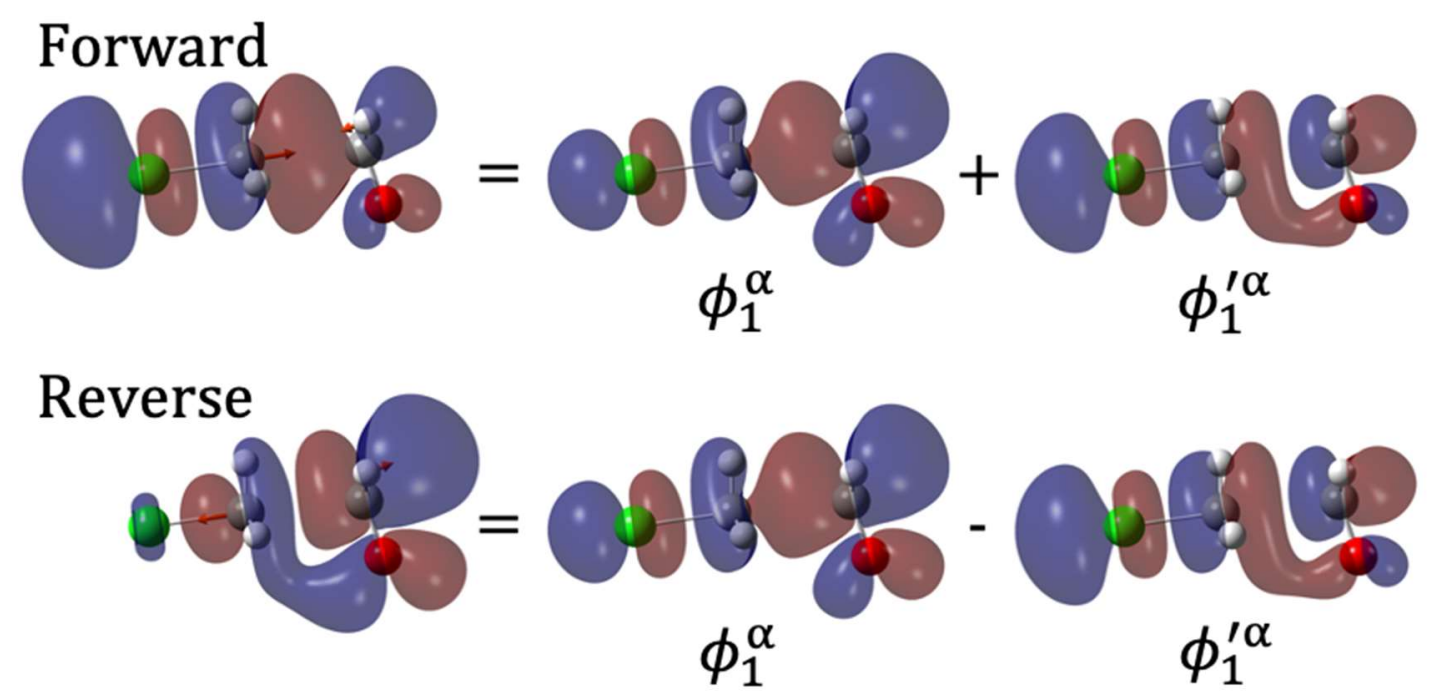

Figure 5. The orbital mixing of occupied and virtual NRO pairs $\left(\phi_{1}^{\alpha}\right.$ and $\left.\phi_{1}^{\prime \alpha}\right)$ with the maximum singular value for nuclear displacements in the directions toward the product (forward) and the reactant (reverse) along the imaginary frequency mode at the electron transfer transition state of the $\mathrm{H}_{2} \mathrm{CO}^{-}+$ $\mathrm{CH}_{3} \mathrm{Cl}$ reaction. In the forward direction, the NRO pair $\left(\phi_{1}^{\alpha}\right.$ and $\left.\phi_{1}^{\prime \alpha}\right)$ mixes with the same sign, indicating the electron transfer from formaldehyde to methyl chloride. In the reverse direction, on the other hand, the NRO pairs mix with opposite signs, indicating the electron transfer from methyl chloride to formaldehyde.

The NROs with non-zero singular values have the point-group irreducible representations for the nuclear displacement. In this reaction, the reaction complex has $C_{s}$ symmetry at the ET-TS structure and the imaginary frequency mode belongs to the $\mathrm{A}^{\prime}$ representation. Due to the $C_{s}$ symmetry, all NROs with non-zero singular values belong to either the irreducible representation of A' or A". Similar to the selection rule for giving non-zero transition amplitudes in the case of electronic excitations, the direct product of the irreducible representations of the occupied NRO, virtual NRO, and nuclear displacements must be totally symmetric. Considering the nuclear displacement (A') of the imaginary frequency mode at ET-TS, the only allowed combinations for the symmetries of the NRO pairs are 
$\left(\mathrm{A}^{\prime}, \mathrm{A}^{\prime}\right)$ and $\left(\mathrm{A}^{\prime \prime}, \mathrm{A}^{\prime \prime}\right)$. Another example is presented for the symmetry of NROs in Fig. A3 of APPENDIX.

For analyzing the effect of occupied orbital-virtual orbital mixing based on the electron density, the first-order one-electron density variation is expressed using CMOs as: ${ }^{25}$

$$
\rho_{\mathrm{VO}}^{(1)}=\sum_{i, a} \mathrm{U}_{a i}^{(1) *} \psi_{a}^{*} \psi_{i}+\mathrm{U}_{a i}^{(1)} \psi_{i}^{*} \psi_{a}
$$

Using the NROs, this equation is represented as

$$
\rho_{\mathrm{VO}}^{(1)}=\sum_{i} n_{i} \lambda_{i}\left(\phi_{i}^{\prime *} \phi_{i}+\phi_{i}^{\prime} \phi_{i}^{*}\right)
$$

This equation simplifies the feature of the one-electron density variation. For the real part of the product of the NRO pair, the one-electron density increases in the in-phase region and decreases in the anti-phase region. Figure 6 shows the product of the most contributing NRO pair for the forward and reverse directions of the IRC at ET-TS, displaying the increasing (yellow) and decreasing (cyan) regions of the one-electron density. Note that the sign of the one-electron density variation is opposite for the forward or reverse directions, because the phase of each NRO pair becomes opposite for the reverse nuclear displacement direction (see Sec. II). Figure 6 also indicates that the electron transfer proceeds from $\pi^{*} \mathrm{CO}$ of the formaldehyde anion to $\sigma^{*}{ }_{\mathrm{C}-\mathrm{Cl}}$ of methyl chloride in the forward reaction and the opposite electron transfer progresses in the reverse reaction, as expected from Fig. 5. As described in Sec. II, reversing the phase of one orbital in the NRO pair turns over the phase of another orbital, in definition. Therefore, because the phase reversal of the NRO pair is carried out as $\left(\phi_{i}, \phi_{i}^{\prime}\right) \rightarrow\left(-\phi_{i},-\phi_{i}^{\prime}\right)$, the sign of $\rho_{\mathrm{VO}}^{(1)}$ in Eq. (22) is always kept invariant. More generally, $\rho_{\mathrm{VO}}^{(1)}$ is invariant for the phase rotation of the NRO pair such as $\left(\phi_{i}, \phi_{i}^{\prime}\right) \rightarrow\left(\phi_{i} e^{i \theta}, \phi_{i}^{\prime} e^{i \theta}\right)$, where $\theta$ denotes arbitrary phase angle, and therefore, $\rho_{\mathrm{VO}}^{(1)}$ is uniquely determined for any nuclear displacement. The discussion in Fig. 5 is also not affected by the phase reversal of the NROs. This consistency comes 
from the nature of the $\mathrm{U}$ matrix, which is a solution of the CPSCF equation. Note that as different from the CMOs, NROs implicitly contain information about the nuclear displacement through the U matrix of the CPSCF equation. The simple relationship with the one-electron density variation under nuclear displacement is one of the most important features of the NROs.

\section{Forward}

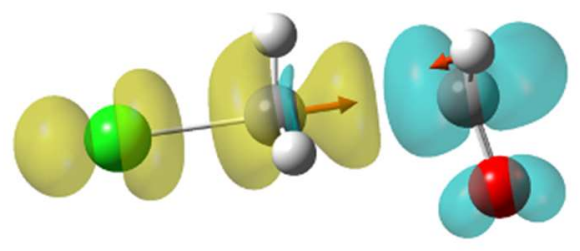

\section{Reverse}

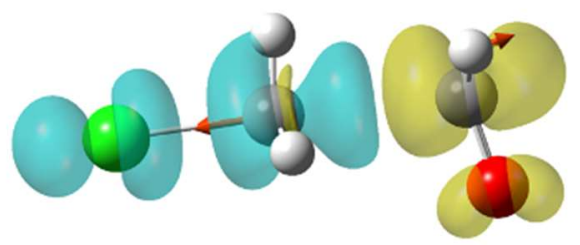

Figure 6. Images of products of the NRO pair $\left(\phi_{1}^{\alpha}\right.$ and $\left.\phi_{1}^{\prime \alpha}\right)$ with the maximum singular value for the forward and reverse nuclear displacements of the imaginary frequency mode, indicated by orange vector arrows, at the electron transfer transition state of the $\mathrm{H}_{2} \mathrm{CO}^{-}+\mathrm{CH}_{3} \mathrm{Cl}$ reaction. The increase and decrease of the one-electron density are shown as yellow and cyan regions, respectively.

Let us next focus on the singular value of the $\mathrm{U}$ matrix corresponding to the NRO pair at the structures on the IRC. Figure 7 displays the sum of the squared singular values, $\lambda^{2}$, of the $\mathbf{U}_{\text {vo }}^{(1)}$ matrix in Eq. (17) for $\alpha$ - and $\beta$-spin orbitals on the IRC. The figure clearly shows that the sum of $\lambda^{2}$ gives the maximum sharp peak at the TS structure for the $\alpha$-spin orbital, indicating that $\alpha$-spin electron transfer mainly proceeds at the TS structure. This result suggests that the singular value of the $\mathbf{U}_{\mathrm{VO}}^{(1)}$ matrix explains the physical meaning of the TS structure from the viewpoint of the electronic theory. The figure also shows that the sum of $\lambda^{2}$ also provides another sharp peak for the $\beta$-spin orbital at the shoulder of the potential energy $\left(s=3.43 \mathrm{amu}^{1 / 2} \mathrm{bohr}\right)$, indicating that $\beta$-spin electron transfer proceeds after the main $\alpha$-spin electron transfer. 


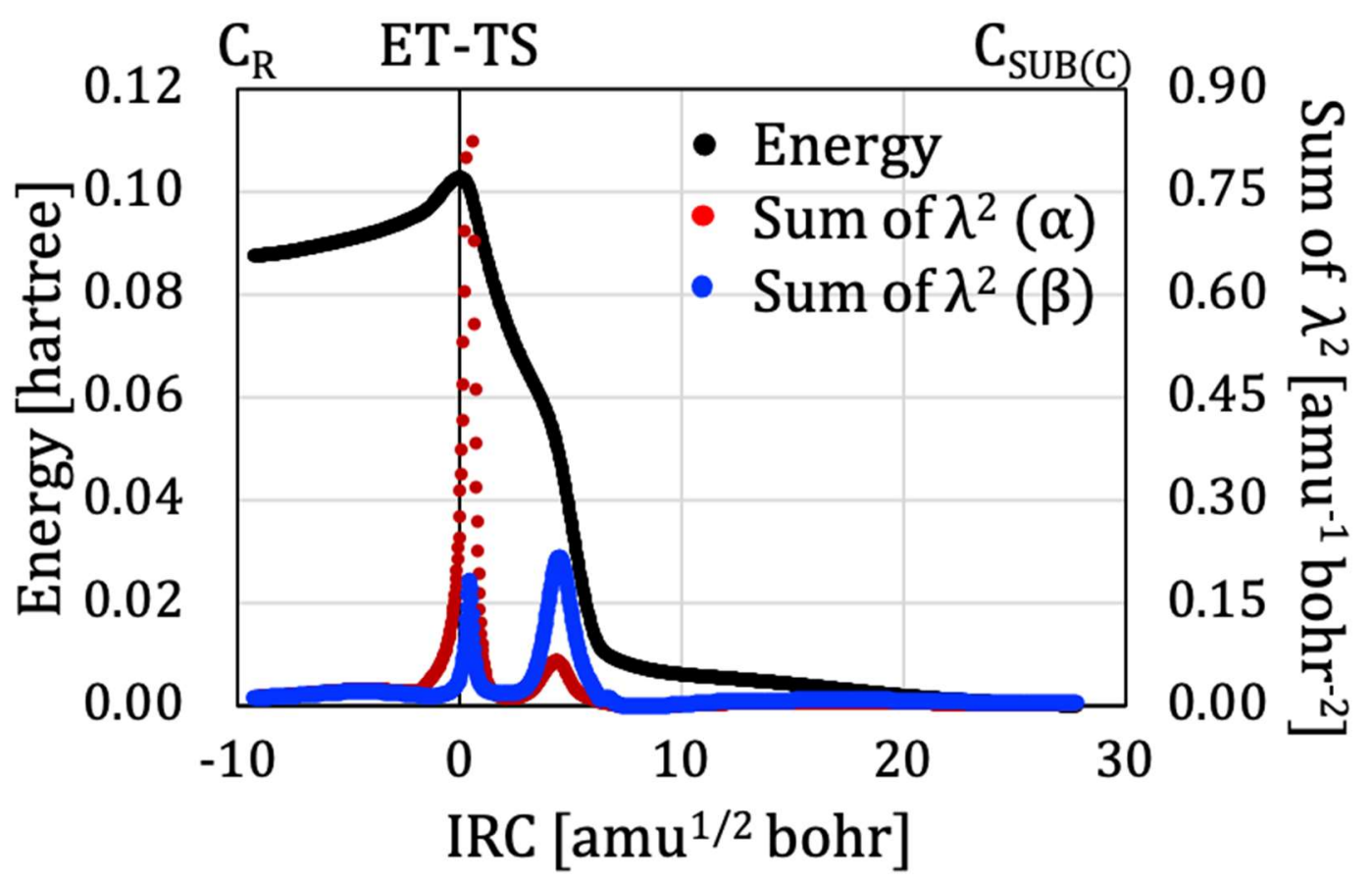

Figure 7. The change in potential energy along the IRC of the $\mathrm{H}_{2} \mathrm{CO}^{-}+\mathrm{CH}_{3} \mathrm{Cl}$ reaction, for which the energy of the product, $\mathrm{C}_{\mathrm{SUB}(\mathrm{C})}$, is set to be zero, and the change in the sum of squared singular values, $\lambda^{2}$, of $\mathbf{U}_{\mathrm{vO}}^{(1)}$ for $\alpha$ - and $\beta$-spin orbitals. The first peak of the sum of $\lambda^{2}$ near the electron transfer transition state mainly corresponds to the $\alpha$-NRO pair of Fig. 3 and the second peak at the shoulder of the potential energy ( $3.43 \mathrm{amu}^{1 / 2}$ bohr) corresponds to the $\beta$-NRO pair.

Figure 8 displays the dominant NROs at the two sharp peaks of the sum of squared singular values, i.e., ET-TS and the shoulder of the potential, with the valence bond configuration mixing (VBCM) diagram of this reaction by Shaik et al. ${ }^{32-35}$. According to the VBCM diagram, the $\pi^{*}$-SOMO of formaldehyde anion $\left(\pi^{*} \mathrm{CO}\right)$ and the $\sigma^{*}$-LUMO of methyl chloride $\left(\sigma^{*} \mathrm{C}-\mathrm{Cl}\right)$ play an important role in the electron transfer at ET-TS. ${ }^{32}$ Note that the contribution of $\pi^{*} \mathrm{CO}$ and $\sigma^{*} \mathrm{C}-\mathrm{Cl}$ is not easily derived from the CMOs due to the highly-delocalized nature of the virtual CMOs in Fig. 2. As consistent with 
the VBCM analysis, ${ }^{34}$ the product of the dominant NRO, $\phi_{1}^{\alpha} \phi_{1}^{\prime \alpha}$, indicates that electron transfer from $\pi^{*} \mathrm{CO}$ to $\sigma^{*} \mathrm{C}$-Cl occurs at ET-TS. The product of the dominant $\beta$-spin NROs indicates the electron transfer from the methyl group to the chlorine atom, which may contribute to the dissociation of the chlorine anion, at ET-TS, though the contribution is small for the mixing of $\beta$-spin NROs. At the shoulder, the $\phi_{1}^{\alpha} \phi_{1}^{\prime \alpha}$ indicates that the $\alpha$-electron transfer proceeds from the non-bonding $\sigma^{*}{ }_{\mathrm{C}-\mathrm{Cl}}$ (or $\mathrm{n}_{\mathrm{C}}$ ) orbital of the methyl radical to the $\pi^{*} \mathrm{CO}$ orbital of $\mathrm{H}_{2} \mathrm{CO}$. On the other hand, the product of the dominant NROs of the $\beta$-spin electron indicates the electron transfer from $\pi_{\mathrm{CO}}$ to $\sigma^{*} \mathrm{C}$-Cl orbitals, resulting in the formation of a $\mathrm{C}-\mathrm{C} \sigma$ bond. According to the NRO results, the electron transfers proceeding at ET-TS and at the potential shoulder induce the $\mathrm{C}-\mathrm{Cl}$ bond dissociation by the electron transfer from $\mathrm{H}_{2} \mathrm{CO}^{-}$to $\mathrm{H}_{3} \mathrm{CCl}$ and the formation of the $\mathrm{C}-\mathrm{C} \sigma$ bond by the $\mathrm{CH}_{3}$ radical attacking $\mathrm{H}_{2} \mathrm{CO}$, respectively. Since these electron transfers are consistent with that of the above-mentioned VBCM diagram, ${ }^{34}$ it is concluded that the NRO pair correctly represents the reaction mechanism based on the electronic theory. Note that the reaction analysis based only on the energy profile may incorrectly interpret this reaction as a concerted $\mathrm{S}_{\mathrm{N}} 2$ reaction. Therefore, we suggest that the NRO-based reaction analysis method will play a powerful role in future reaction analyses. 


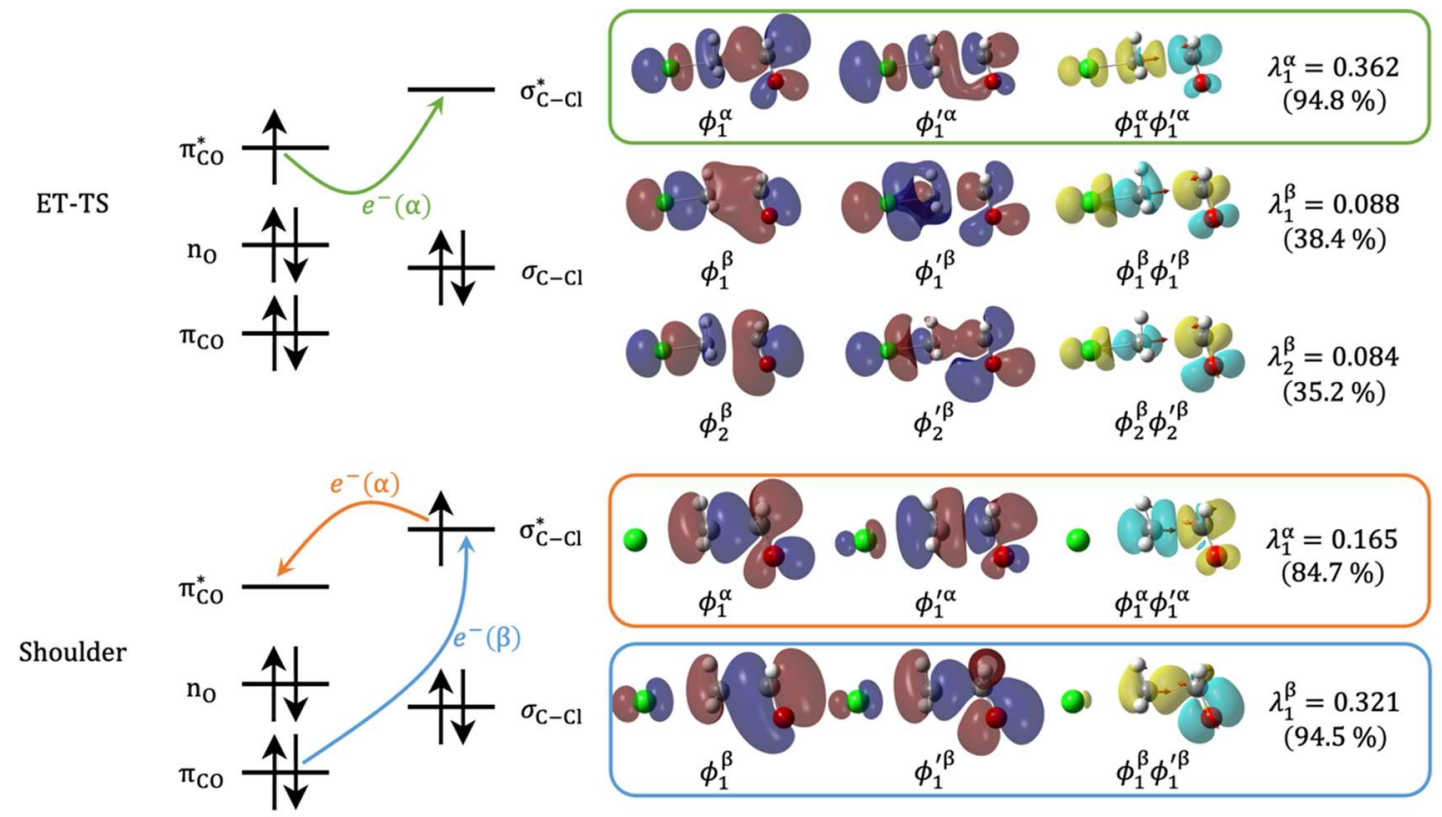

Figure 8. The dominant natural reaction orbitals (NROs) at the two sharp peaks of the sum of squared singular values, the electron transfer transition state (ET-TS) and the shoulder of the potential at 3.43 $\mathrm{amu}^{1 / 2}$ bohr on the IRC in Fig. 7, with the valence bond configuration mixing diagram of this reaction in Ref. 32. Images of products of the NRO pairs are also illustrated with the vector arrows corresponding to the nuclear displacements. The yellow and cyan regions show the increase and decrease of the one-electron density, respectively. The singular values and contribution ratios for the dominant NRO pairs are shown at the rightmost part.

Finally, let us apply the NROs to the reactions reported by Nouri and Tantillo, ${ }^{48}$ which includes multiple bond rearrangement but contains no shoulder features in the energy profile. These NRO images are illustrated for the carbocation rearrangement of cyclohexyl cation to methylcyclopentyl cation with the energy profile and the squared singular values in Fig. $\mathbf{S 3}$ of the supporting information. In addition, those of Diels-Alder reaction between butadiene and nitroethylene (Fig. S1), Schmidt-Aubé reaction (Fig. S2), Claisen rearrangement (Fig. S4), and proton 
transfer reaction of malonaldehyde (Fig. S5), are also displayed in the supporting information. As shown in Fig. S3-1, no shoulder is given in the potential energy at the position where the sum of the squared singular value has a peak, even though this reaction contains dual bond rearrangement. This indicates that the NROs are much more sensitive than the potential energy surface for describing the reaction mechanism. Actually, the NROs with the maximum singular values in Figs. S3-2 and S3-3 correctly represent the $\mathrm{C}-\mathrm{H}$ bond cleavage and 1,2-hydride shift required to proceed with this rearrangement reaction, respectively, though the 1,2-hydride shift is not detected in the potential energy profile. For other reactions included in the supporting information, it is confirmed that the NROs can reasonably explain the reaction mechanisms from the viewpoint of the electronic theory. We, therefore, conclude that the NROs can provide more sophisticated chemical reaction mechanisms than those of the energy profiles. 


\section{CONCLUDING REMARKS}

In this study, we propose natural reaction orbital (NRO) as a sophisticated tool for analyzing chemical reactions from the viewpoint of the electronic theory. The NRO-based reaction analysis method has five distinctive advantages for availability and applicability:

1. The NRO pair that drives the chemical reaction is automatically extracted based on the nuclear coordinate derivative of canonical molecular orbitals by solving the CPSCF equation.

2. The delocalization nature of virtual canonical orbitals, which complicates orbital-based reaction analysis, is dramatically improved in virtual NROs.

3. Since the NROs can be calculated at any given molecular structure, they reduce the effort for analyzing significant electron transfers in chemical reaction paths.

4. NROs are robust due to their low dependence on the basis set (see Figs. A1 and A2 in APPENDIX).

5. Under the nuclear displacement perturbation in the point group symmetry, the NROs transform in the irreducible representation of this point group (see Fig. A3 in APPENDIX). NRO pairs give non-zero singular values only if the direct product of the irreducible representations of nuclear displacements, occupied NRO, and virtual NRO is totally symmetric (see Figs. A3 and A4 in APPENDIX).

Note that the NRO-based reaction analysis is the only method that satisfies all of these advantages among similar orbital-based analysis methods. The NROs are obtained by solving the CPSCF equation for the perturbation of the nuclear displacement on the IRC that is considered in the WoodwardHoffmann rule and the frontier orbital theory.

Applying the NRO-based analysis method to the $\mathrm{H}_{2} \mathrm{CO}^{-}+\mathrm{H}_{3} \mathrm{CCl}$ reaction, it is found that the sum of the squared singular values provides the maximum sharp peak at the transition state structure, indicating the $\mathrm{C}-\mathrm{Cl}$ bond dissociation by the electron transfer from $\mathrm{H}_{2} \mathrm{CO}^{-}$to $\mathrm{H}_{3} \mathrm{CCl}$. It is also found that another sharp peak is provided at the shoulder of the potential energy, which 
corresponds to the formation of the $\mathrm{C}-\mathrm{C} \sigma$ bond by the $\mathrm{CH}_{3}$ radical attacking $\mathrm{H}_{2} \mathrm{CO}$. The NRO-based analysis method is also applied to five reactions including multiple bond rearrangement (see Supporting Information). As a result, it is found that this analysis also provides a peak of the sum of $\lambda^{2}$ at a reaction coordinate where no shoulders are given for the potential energy profile. This indicates that the NROs are much more sensitive than the potential energy surface for representing reaction mechanisms. In summary, it is confirmed that the NRO-based reaction analysis method has universality and robustness, and it is a powerful tool for describing sophisticated reaction mechanisms.

\section{ACKNOWLEDGEMENT}

SE thanks the Institute for Quantum Chemical Exploration through the Research Fellowship for Young Scientists and the MEXT Doctoral program for DataRelated InnoVation Expert Hokkaido University (D-DRIVE-HU). This work was also partly supported by the Elements Strategy Initiative of MEXT (JPMXP0112101003), the Photoexcitonix Project at Hokkaido University, and JST CREST, Japan (JPMJCR1902). A part of calculations was performed using the Research Center for Computational Science, Okazaki, Japan.

\section{DATA AVAILABILITY}

The data that supports the findings of this study are available within the article [and its supplementary material].

\section{SUPPORTING INFORMATION}

NRO analysis performed along the IRC for five different types of chemical reactions: (1) Diels-Alder reaction between butadiene and nitroethylene, (2) Schmidt-Aubé reaction, (3) carbocation rearrangement, (4) Claisen rearrangement, and (5) proton transfer in malonaldehyde. 

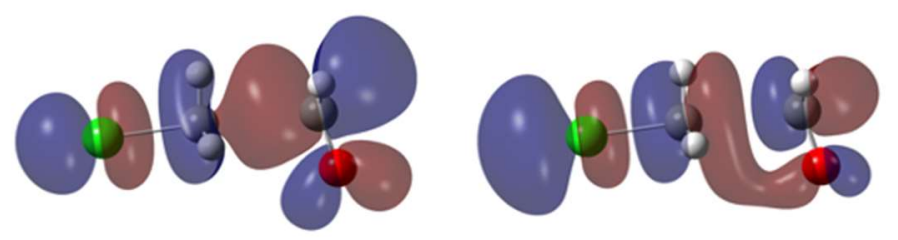

$$
\lambda_{1}(\alpha)=0.362
$$

$\phi_{1}^{\alpha}$

$\phi_{1}^{\prime \alpha}$
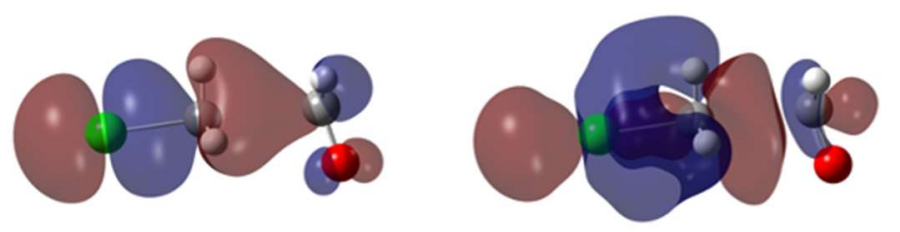

$\lambda_{2}(\alpha)=0.056$

$\phi_{2}^{\alpha}$

$\phi_{2}^{\prime \alpha}$
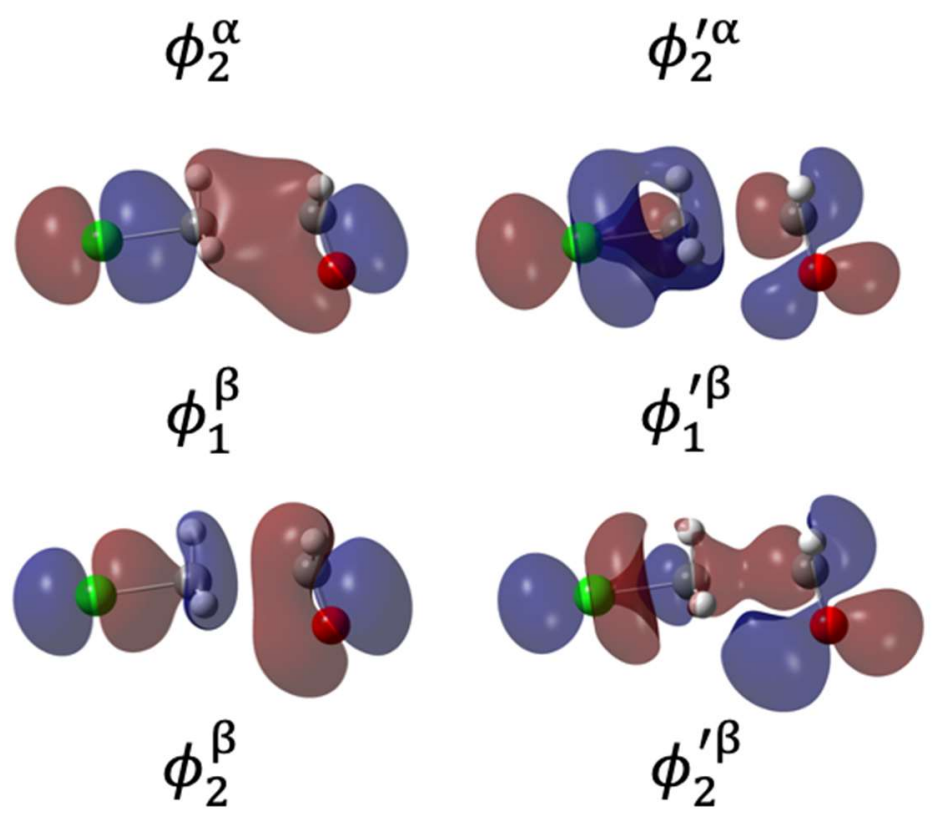

$\lambda_{1}(\beta)=0.088$

$\phi_{1}^{\beta}$

$\phi_{1}^{\prime \beta}$
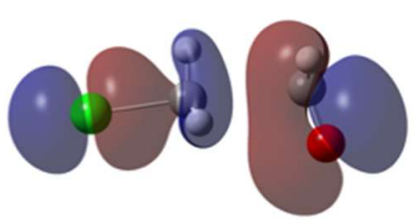

$\phi_{2}^{\beta}$

\section{$\lambda_{2}(\beta)=0.084$}

Figure A1. Natural reaction orbitals (NROs) at the electron transfer transition state of $\mathrm{H}_{2} \mathrm{CO}^{-}+\mathrm{CH}_{3} \mathrm{Cl}$ reaction with the imaginary frequency mode toward the product side as the nuclear displacement direction, calculated at the UHF/6-31+G(d,p) level. For the $\alpha$ spin and $\beta$ spin orbitals, two pairs of occupied and virtual NROs with the largest and second largest singular values, respectively, are shown. The dominant NRO pair is $\phi_{1}^{\alpha}$ and $\phi_{1}^{\prime \alpha}$. 

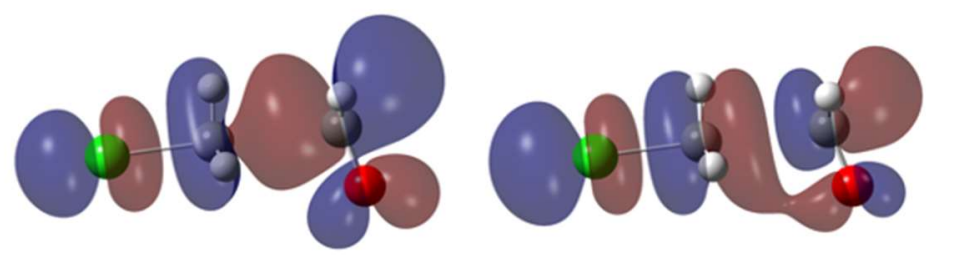

\section{$\lambda_{1}(\alpha)=0.387$}
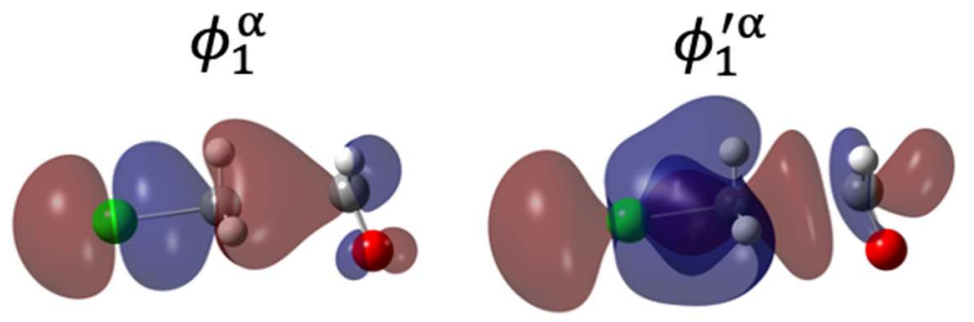

$$
\lambda_{2}(\alpha)=0.067
$$
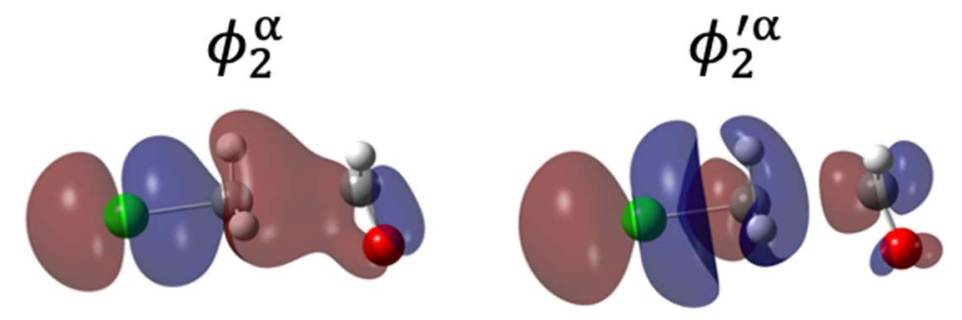

$$
\lambda_{1}(\beta)=0.096
$$
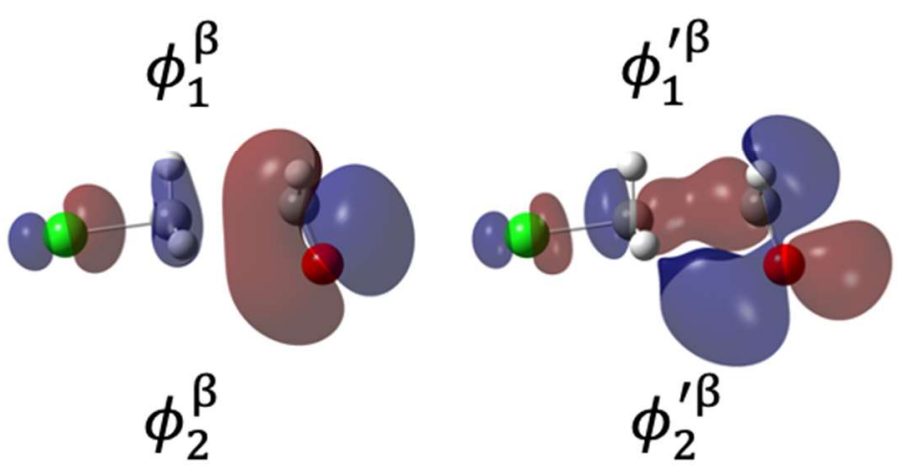

\section{$\lambda_{2}(\beta)=0.084$}

$$
\phi_{2}^{\beta}
$$

$\phi_{2}^{\prime \beta}$

Figure A2. Natural reaction orbitals (NROs) at the electron transfer transition state of $\mathrm{H}_{2} \mathrm{CO}^{-}+\mathrm{CH}_{3} \mathrm{Cl}$ reaction with the imaginary frequency mode toward the product side as the nuclear displacement direction, calculated at the UHF/aug-cc-pVTZ level. For the $\alpha$ spin and $\beta$ spin orbitals, two pairs of occupied and virtual NROs with the largest and second largest singular values, respectively, are shown. These NROs are almost the same as those obtained with the 6-31+G(d,p) basis set (Fig. A1). This result indicates that the basis set dependence of the NRO is sufficiently small. 


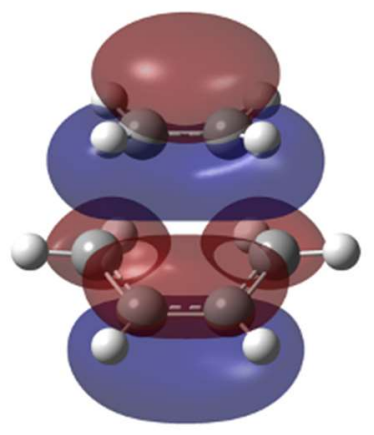

$\phi_{1}\left(\mathrm{~A}^{\prime}\right)$

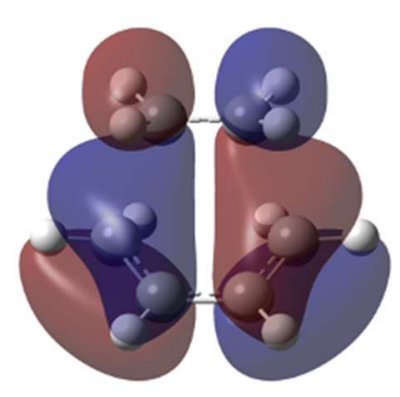

$\phi_{2}\left(\mathrm{~A}^{\prime \prime}\right)$

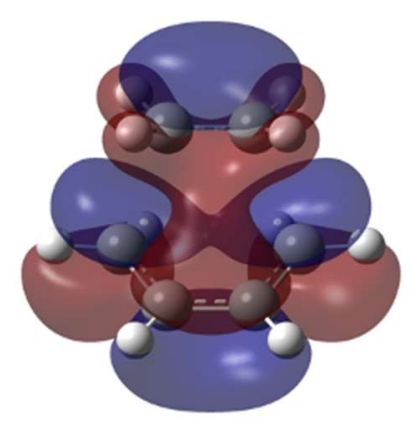

$\phi_{1}^{\prime}\left(\mathrm{A}^{\prime}\right)$

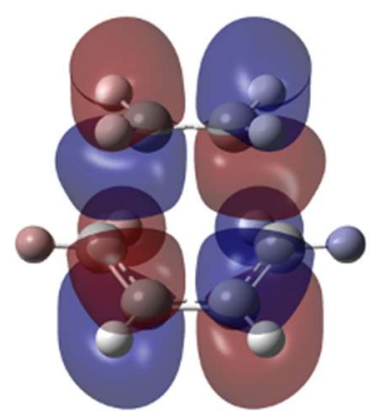

$\phi_{2}^{\prime}\left(\mathrm{A}^{\prime \prime}\right)$

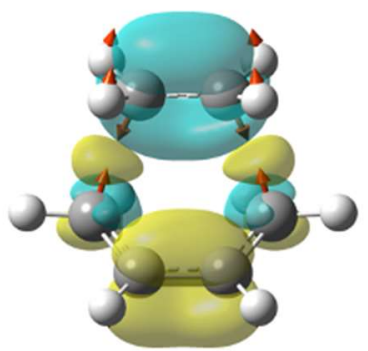

$\phi_{1}^{\prime} \phi_{1}\left(\mathrm{~A}^{\prime}\right)$

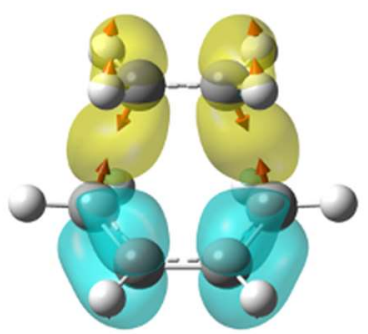

$\phi_{2}^{\prime} \phi_{2}\left(\mathrm{~A}^{\prime}\right)$

Figure A3. Natural reaction orbitals (NRO) pairs with the maximum singular value ( $\phi_{1}$ and $\left.\phi_{1}^{\prime}\right)$ and the second maximum singular value $\left(\phi_{2}\right.$ and $\left.\phi_{2}^{\prime}\right)$ calculated at the transition state $\left(\mathrm{C}_{\mathrm{s}}\right.$ point group) with the imaginary frequency mode of $\mathrm{A}^{\prime}$ symmetry in the Diels-Alder reaction of ethylene and 1,3butadiene at the RHF/6-31G(d,p) level. The product of the NROs is also shown for each pair of occupied NRO and virtual NRO. The singular value of the $\left(\phi_{1}, \phi_{1}^{\prime}\right)$ pair is $0.422 \mathrm{amu}^{-1 / 2} \mathrm{bohr}^{-1}$ (contribution: $51.3 \%$ ), and that of the $\left(\phi_{2}, \phi_{2}^{\prime}\right)$ pair is $0.391 \mathrm{amu}^{-1 / 2} \mathrm{bohr}^{-1}$ (contribution: $43.9 \%$ ). Thus, these two sets of NROs cover $95.2 \%$ of the description of orbital mixing at TS. 

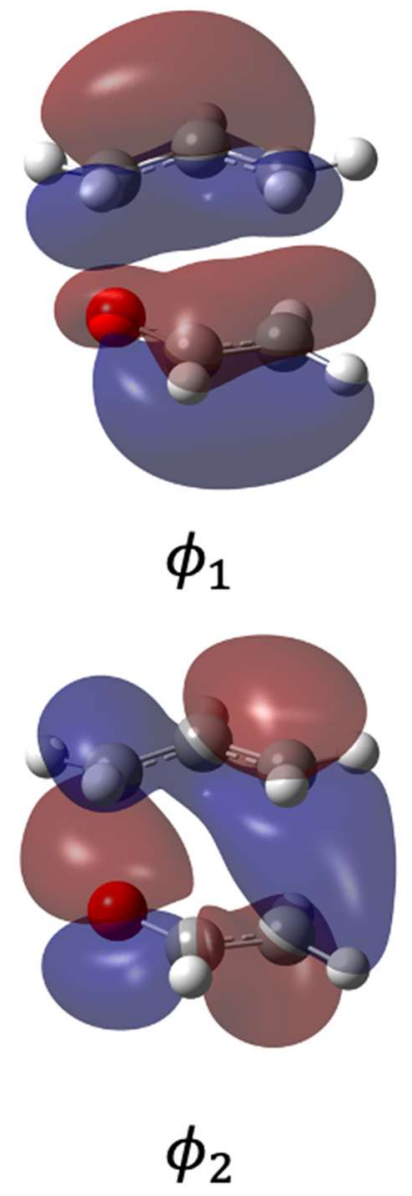
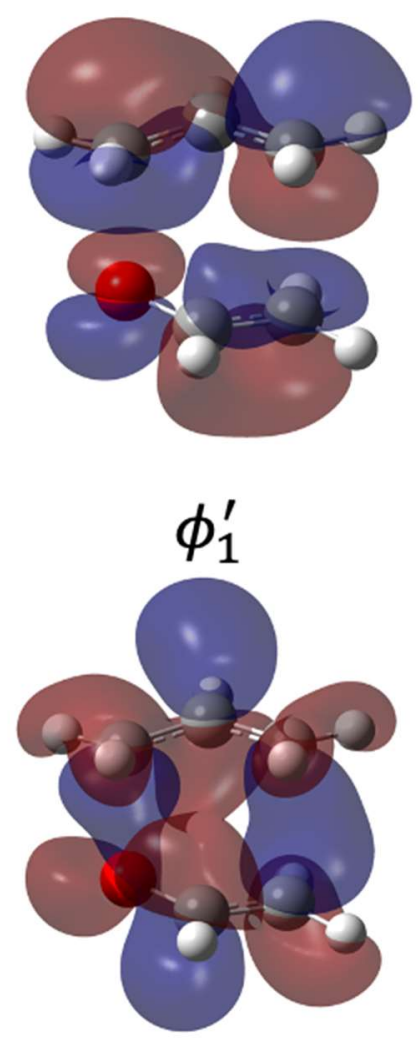

$\phi_{2}^{\prime}$

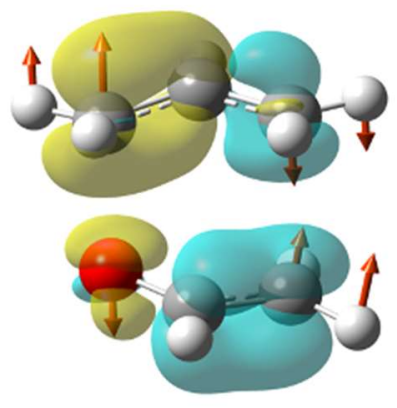

$\phi_{1}^{\prime} \phi_{1}$

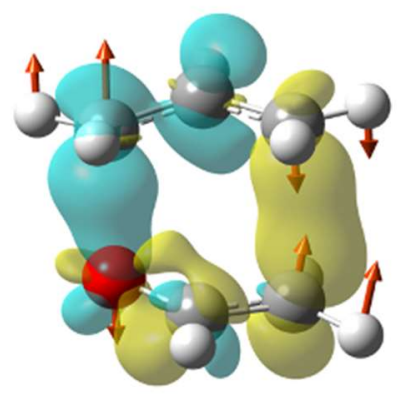

$\phi_{2}^{\prime} \phi_{2}$

Figure A4. Natural reaction orbitals (NRO) pairs with the maximum singular value ( $\phi_{1}$ and $\left.\phi_{1}^{\prime}\right)$ and the second maximum singular value $\left(\phi_{2}\right.$ and $\left.\phi_{2}^{\prime}\right)$ calculated at the transition state with the imaginary frequency mode in the Claisen rearrangement of allyl vinyl ether at the B3LYP/6-311+G(d,p) level. The product of the NROs is also shown for each pair of occupied NRO and virtual NRO. The singular value of the $\left(\phi_{1}, \phi_{1}^{\prime}\right)$ pair is $0.228 \mathrm{amu}^{-1 / 2}$ bohr-1 $^{-1}$ (contribution: $52.0 \%$ ), and that of the $\left(\phi_{2}, \phi_{2}^{\prime}\right)$ pair is $0.187 \mathrm{amu}^{-1 / 2} \mathrm{bohr}^{-1}$ (contribution: $35.1 \%$ ). Thus, these two sets of NROs cover $87.1 \%$ of the description of orbital mixing at TS. 


\section{REFERENCES}

${ }^{1}$ K. Ohno, H. Mutoh, and Y. Harada, J. Am. Chem. Soc. 105, 4555 (1983).

${ }^{2}$ M. Yamazaki, T. Horio, N. Kishimoto, and K. Ohno, Phys. Rev. A 75, 032721 (2007).

${ }^{3}$ E. Weigold and I.E. McCarthy, Electron Momentum Spectroscopy (Springer US, Boston, MA, 1999).

${ }^{4}$ J. Itatani, J. Levesque, D. Zeidler, H. Niikura, H. Pépin, J.C. Kieffer, P.B. Corkum, and D.M. Villeneuve, Nature 432, 867 (2004).

${ }^{5}$ P. Puschnig, E.-M. Reinisch, T. Ules, G. Koller, S. Soubatch, M. Ostler, L. Romaner, F.S. Tautz, C. Ambrosch-Draxl, and M.G. Ramsey, Phys. Rev. B 84, 235427 (2011).

${ }^{6}$ J.P. Perdew, R.G. Parr, M. Levy, and J.L. Balduz, Phys. Rev. Lett. 49, 1691 (1982).

${ }^{7}$ H. Iikura, T. Tsuneda, T. Yanai, and K. Hirao, J. Chem. Phys. 115, 3540 (2001).

${ }^{8}$ L.J. Sham and M. Schlüter, Phys. Rev. B 32, 3883 (1985).

${ }^{9}$ T. Tsuneda, J.W. Song, S. Suzuki, and K. Hirao, J. Chem. Phys. 133, (2010).

${ }^{10}$ K. Fukui, T. Yonezawa, and H. Shingu, J. Chem. Phys. 20, 722 (1952).

${ }^{11}$ K. Fukui, T. Yonezawa, and C. Nagata, Bull. Chem. Soc. Jpn. 27, 423 (1954).

${ }^{12}$ R.B. Woodward and R. Hoffmann, J. Am. Chem. Soc. 87, 395 (1965).

${ }^{13}$ R. Hoffmann and R.B. Woodward, J. Am. Chem. Soc. 87, 2046 (1965).

${ }^{14}$ R. Hoffmann and R.B. Woodward, J. Am. Chem. Soc. 87, 4388 (1965).

${ }^{15}$ R.B. Woodward and R. Hoffmann, Angew. Chemie Int. Ed. English 8, 781 (1969).

${ }^{16}$ R. Hoffmann and R.B. Woodward, Science 167, 825 (1970).

${ }^{17}$ P. Geerlings, F. De Proft, and W. Langenaeker, Chem. Rev. 103, 1793 (2003).

${ }^{18}$ T. Tsuneda and R.K. Singh, J. Comput. Chem. 35, 1093 (2014).

19 T. Tsuneda, R.K. Singh, and P.K. Chattaraj, Phys. Chem. Chem. Phys. 20, 14211 (2018).

${ }^{20}$ A. E. Reed, F. Weinhold, J. Chem. Phys. 78, 4066 (1983).

${ }^{21}$ E. D. Glendening, C. R. Landis, F.Weinhold, WIREs Comput. Mol. Sci. 2, 1 (2012). 
${ }^{22}$ A. Morita and S. Kato, J. Am. Chem. Soc. 119, 4021 (1997).

${ }^{23}$ R. McWeeny, Rev. Mod. Phys. 32, 335 (1960).

${ }^{24}$ J. Gerratt and I.M. Mills, J. Chem. Phys. 49, 1719 (1968).

${ }^{25}$ J.A. Pople, R. Krishnan, H.B. Schlegel, and J.S. Binkley, Int. J. Quantum Chem. 16, 225 (1979).

${ }^{26}$ M. Frisch, M. Head-Gordon, and J. Pople, Chem. Phys. 141, 189 (1990).

${ }^{27}$ R. Fournier, J. Chem. Phys. 92, 5422 (1990).

${ }^{28}$ R.L. Martin, J. Chem. Phys. 118, 4775 (2003).

${ }^{29}$ J.-X. Zhang, F.K. Sheong, and Z. Lin, Chem. - A Eur. J. 24, 9639 (2018).

${ }^{30}$ F.K. Sheong, J.-X. Zhang, and Z. Lin, Phys. Chem. Chem. Phys. 22, 10076 (2020).

${ }^{31}$ J. Zhang, F.K. Sheong, and Z. Lin, WIREs Comput. Mol. Sci. 10, (2020).

${ }^{32}$ L. Eberson and S.S. Shaik, J. Am. Chem. Soc. 112, 4484 (1990).

${ }^{33}$ G.N. Sastry and S. Shaik, J. Am. Chem. Soc. 117, 3290 (1995).

${ }^{34}$ G.N. Sastry and S. Shaik, J. Phys. Chem. 100, 12241 (1996).

${ }^{35}$ S. Shaik, D. Danovich, G.N. Sastry, P.Y. Ayala, and H.B. Schlegel, J. Am. Chem. Soc. 119, 9237 (1997).

${ }^{36}$ Y. Harabuchi and T. Taketsugu, Theor. Chem. Acc. 130, 305 (2011).

${ }^{37}$ Y. Alexeev, M.W. Schmidt, T.L. Windus, and M.S. Gordon, J. Comput. Chem. 28, 1685 (2007).

${ }^{38}$ K. Fukui, J. Phys. Chem. 74, 4161 (1970).

${ }^{39}$ W.J. Hehre, R. Ditchfield, and J.A. Pople, J. Chem. Phys. 56, 2257 (1972).

${ }^{40}$ G.A. Petersson, A. Bennett, T.G. Tensfeldt, M.A. Al - Laham, W.A. Shirley, and J. Mantzaris, J. Chem. Phys. 89, 2193 (1988).

${ }^{41}$ G.A. Petersson and M.A. Al - Laham, J. Chem. Phys. 94, 6081 (1991).

${ }^{42}$ T. Clark, J. Chandrasekhar, G.W. Spitznagel, and P.V.R. Schleyer, J. Comput. Chem. 4, 294 (1983).

${ }^{43}$ M.J. Frisch, G.W. Trucks, H.B. Schlegel, G.E. Scuseria, M.A. Robb, J.R. Cheeseman, G. Scalmani, V. Barone, G.A. Petersson, H. Nakatsuji, X. Li, M. Caricato, A. V Marenich, J. Bloino, B.G. Janesko, R. 
Gomperts, B. Mennucci, H.P. Hratchian, J. V Ortiz, A.F. Izmaylov, J.L. Sonnenberg, D. Williams-Young, F. Ding, F. Lipparini, F. Egidi, J. Goings, B. Peng, A. Petrone, T. Henderson, D. Ranasinghe, V.G. Zakrzewski, J. Gao, N. Rega, G. Zheng, W. Liang, M. Hada, M. Ehara, K. Toyota, R. Fukuda, J. Hasegawa, M. Ishida, T. Nakajima, Y. Honda, O. Kitao, H. Nakai, T. Vreven, K. Throssell, J. Montgomery, J. A., J.E. Peralta, F. Ogliaro, M.J. Bearpark, J.J. Heyd, E.N. Brothers, K.N. Kudin, V.N. Staroverov, T.A. Keith, R. Kobayashi, J. Normand, K. Raghavachari, A.P. Rendell, J.C. Burant, S.S. Iyengar, J. Tomasi, M. Cossi, J.M. Millam, M. Klene, C. Adamo, R. Cammi, J.W. Ochterski, R.L. Martin, K. Morokuma, O. Farkas, J.B. Foresman, and D.J. Fox, (2016).

${ }^{44}$ T.H. Dunning, J. Chem. Phys. 90, 1007 (1989).

${ }^{45}$ R.A. Kendall, T.H. Dunning, and R.J. Harrison, J. Chem. Phys. 96, 6796 (1992).

${ }^{46}$ D.E. Woon and T.H. Dunning, J. Chem. Phys. 98, 1358 (1993).

${ }^{47}$ S. Sakai, Bull. Chem. Soc. Jpn. 66, 3326 (1993).

${ }^{48}$ D.H. Nouri and D.J. Tantillo, J. Org. Chem. 71, 3686 (2006).

${ }^{49}$ D.J. Tantillo, J. Phys. Org. Chem. 21, 561 (2008). 


\section{Supporting Information}

\section{Natural reaction orbitals}

Shuichi Ebisawa ${ }^{1}$, Masatoshi Hasebe ${ }^{1}$, Takuro Tsutsumi ${ }^{2}$, Takao Tsuneda ${ }^{2,3}$, Tetsuya Taketsugu ${ }^{2,4}$ *

Correspondence to: Tetsuya Taketsugu (E-mail: take@sci.hokudai.ac.jp)

1 Graduate School of Chemical Sciences and Engineering, Hokkaido University, Sapporo 060-0810, Japan

2 Department of Chemistry, Faculty of Science, Hokkaido University, Sapporo 060-0810, Japan

3 Graduate School of Science Technology and Innovation, Kobe University, Nada-ku, Kobe, Hyogo 657-8501, Japan

4 Institute for Chemical Reaction Design and Discovery (WPI-ICReDD), Hokkaido University, Sapporo 001-0021, Japan 

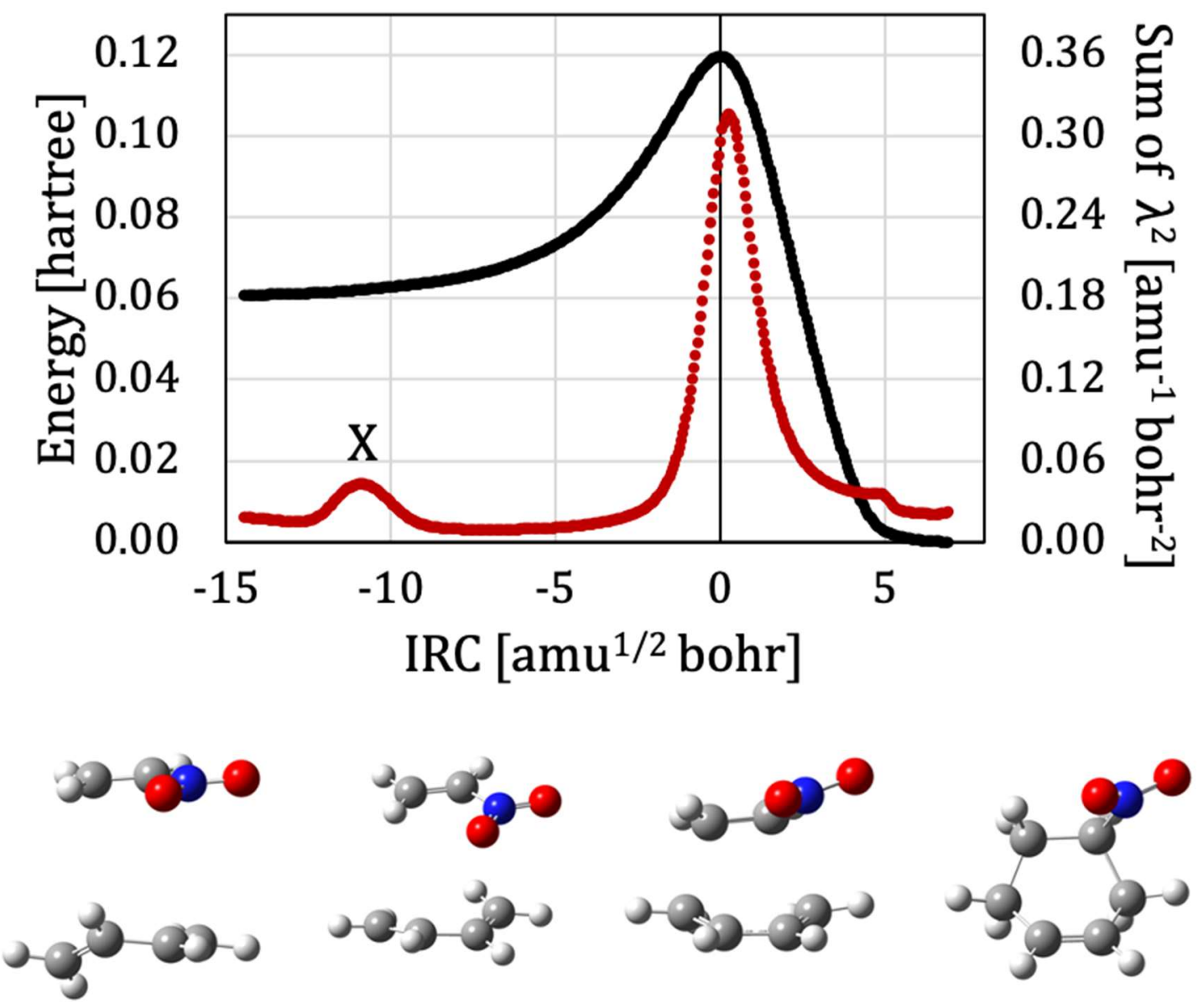

Reactant

$\mathrm{X}$

TS

\section{Product}

Figure S1-1. Change in energy relative to the product (black dots) and sum of squared singular values of $\mathbf{U}_{\mathrm{VO}}^{(1)}$ (red dots) along the IRC of Diels-Alder reaction between butadiene and nitroethylene. The IRC was calculated for the singlet ground state at RHF/6-31G(d,p) level. The structures at the reactant, point X, TS, and product are also shown below the graph. The sum of squared singular values of $\mathbf{U}_{\mathrm{VO}}^{(1)}$ has two peaks: the peak at $-10.90 \mathrm{amu}^{1 / 2}$ bohr (shown by $\mathrm{X}$ ) and the peak near the TS $\left(0.00 \mathrm{amu}^{1 / 2}\right.$ bohr). Though the point $\mathrm{X}$ is not a shoulder of total energy profile, $\mathrm{X}$ is a local maximum of the sum of squared singular values of $\mathbf{U}_{\mathrm{VO}}^{(1)}$. 


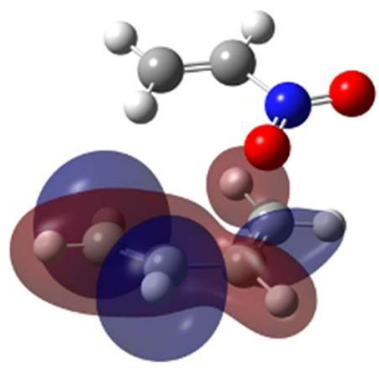

$\phi_{1}$

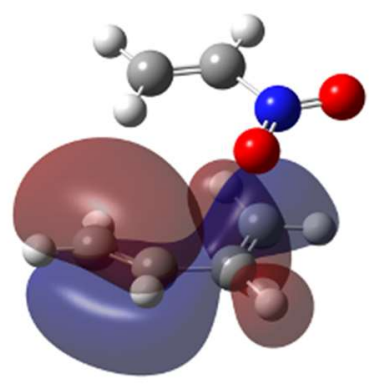

$\phi_{2}$

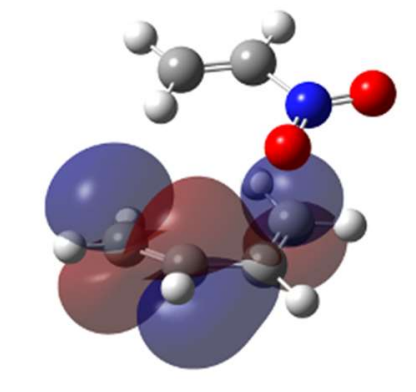

$\phi_{1}^{\prime}$

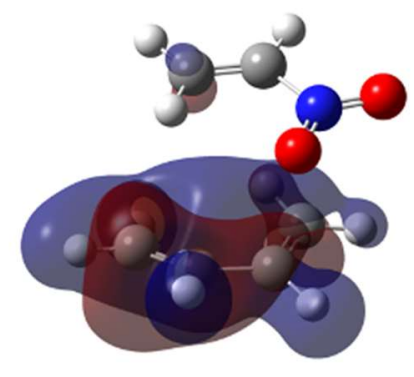

$\phi_{2}^{\prime}$

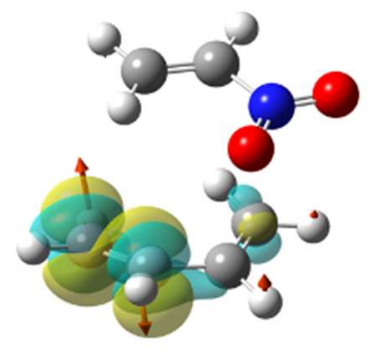

$\phi_{1}^{\prime} \phi_{1}$

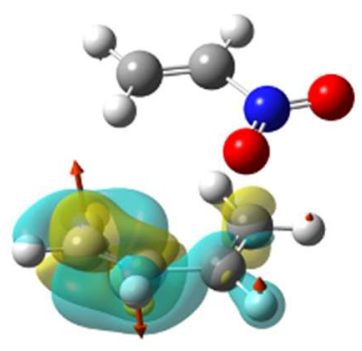

$\phi_{2}^{\prime} \phi_{2}$

Figure S1-2. NRO pairs with the largest and second largest singular values and their products at the point X in Fig. S1-1. The products of the NRO pairs are shown together with the tangent vector of the IRC. The yellow and cyan regions indicate the increase and decrease of the one-electron density, respectively. The singular value and contribution ratio of $\phi_{1}$ and $\phi_{1}^{\prime}$ are $0.152 \mathrm{amu}^{-1 / 2} \mathrm{bohr}^{-1}$ and $53.8 \%$, respectively, while the singular value and contribution ratio of $\phi_{2}$ and $\phi_{2}^{\prime}$ are $0.091 \mathrm{amu}^{-}$ ${ }^{1 / 2} \mathrm{bohr}^{-1}$ and $19.5 \%$, respectively. Thus, these two sets of NROs cover $73.2 \%$ of the virtual-occupied mixing. At the point $\mathrm{X}, \mathrm{NROs}$ are mixed by the twist motion of butadiene. Though the point $\mathrm{X}$ is a local maximum of the sum of squared singular values of $\mathbf{U}_{\mathrm{VO}}^{(1)}$, the density variation caused by the conformational change seems not essential for the Diels-Alder reaction. 


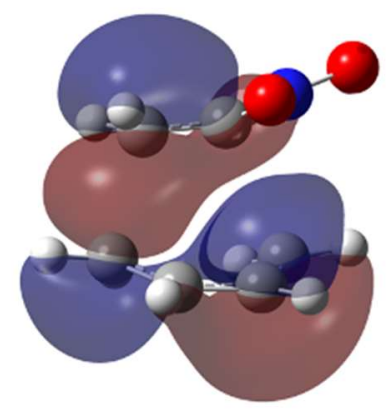

$$
\phi_{1}
$$

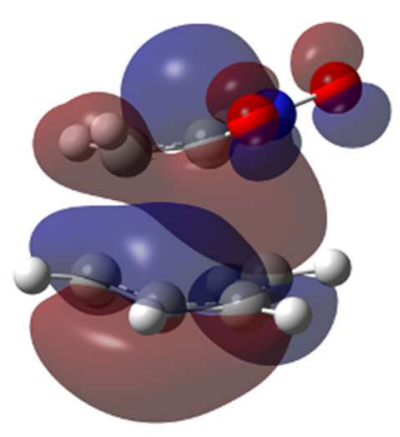

$\phi_{2}$

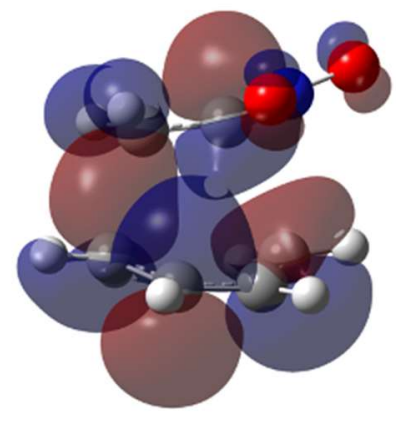

$\phi_{1}^{\prime}$

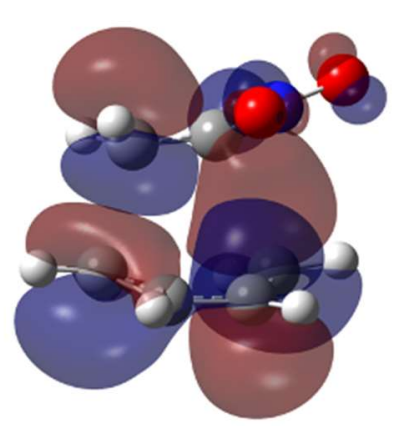

$\phi_{2}^{\prime}$

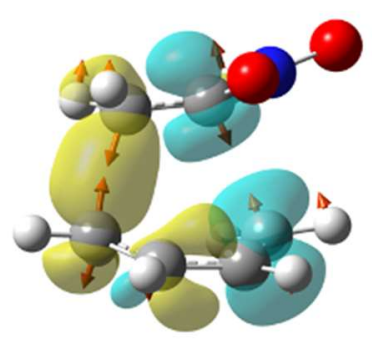

$\phi_{1}^{\prime} \phi_{1}$

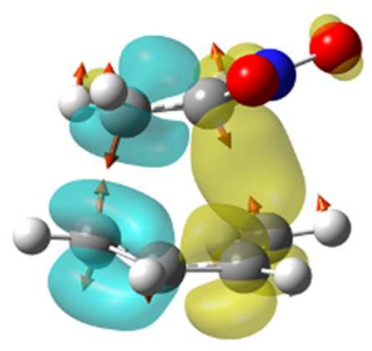

$\phi_{2}^{\prime} \phi_{2}$

Figure S1-3. NRO pairs with the largest and second largest singular values and their products at the TS in Fig. S1-1. The products of the NRO pairs are shown together with the transition vectors with an imaginary frequency. The singular value and contribution ratio of $\phi_{1}$ and $\phi_{1}^{\prime}$ are $0.390 \mathrm{amu}^{-1 / 2}$ bohr $^{-1}$ and $51.3 \%$, respectively, while the singular value and contribution ratio of $\phi_{2}$ and $\phi_{2}^{\prime}$ are $0.357 \mathrm{amu}^{-1 / 2} \mathrm{bohr}^{-1}$ and $43.0 \%$, respectively. Thus, these two sets of NROs cover $94.3 \%$ of the virtual-occupied mixing. The two NRO products clearly show the density increase in the region of two $\mathrm{C}-\mathrm{C} \sigma$ bonds to be formed. A slight density increase in the region of $\mathrm{C} 2-\mathrm{C} 3 \pi$ bond of butadiene is also observed. This density increase corresponds to the formation of $\mathrm{C} 2-\mathrm{C} 3 \pi$ bond. Contrary, electron density decreases in the regions of $\mathrm{C} 1-\mathrm{C} 2 \pi$ bond and $\mathrm{C} 3-\mathrm{C} 4 \pi$ bond, associated with the cleavage of $\mathrm{C}-\mathrm{C} \pi$ bonds. 


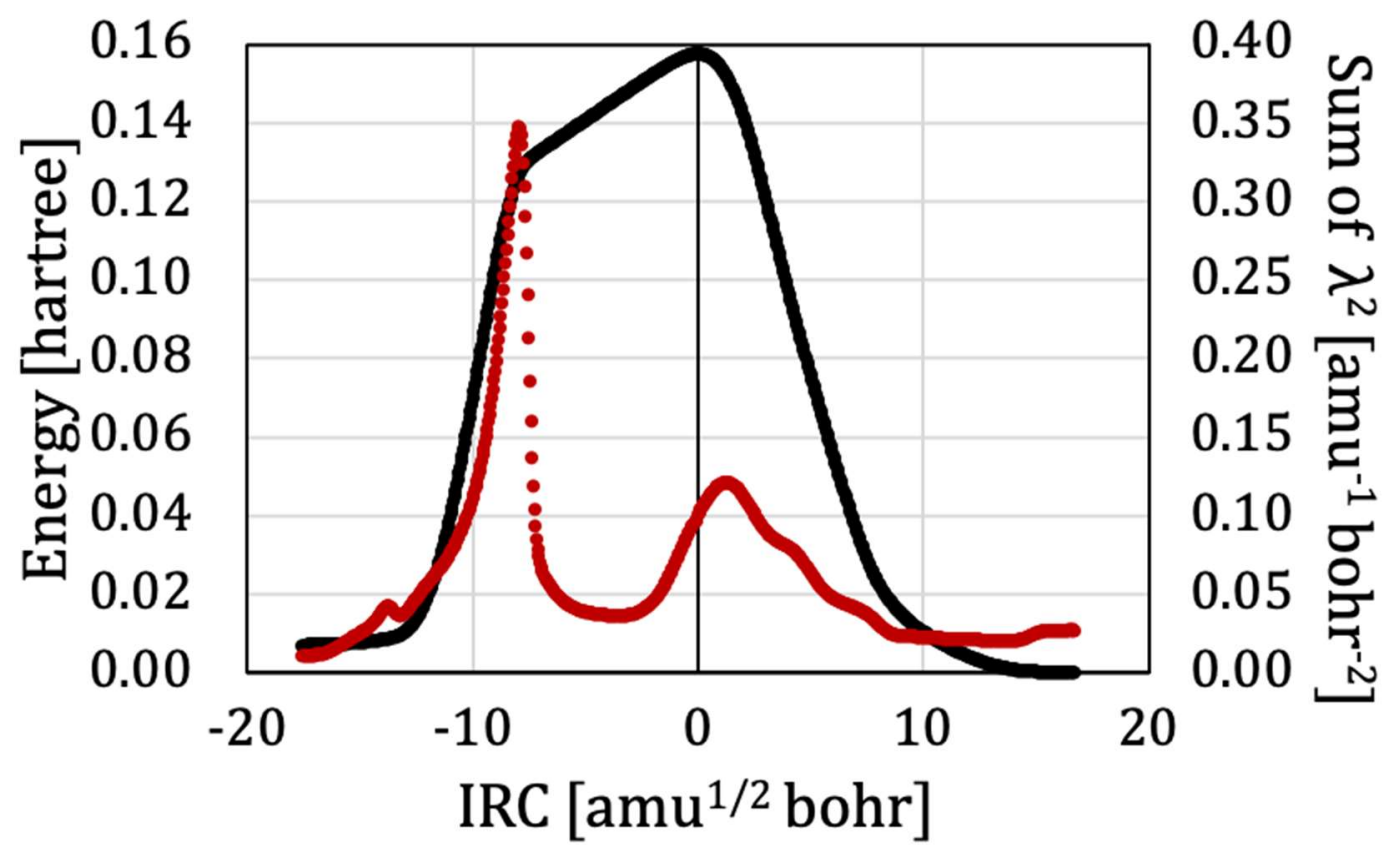

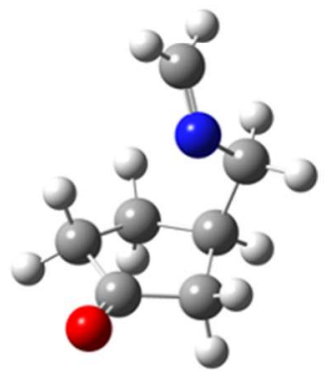

Reactant

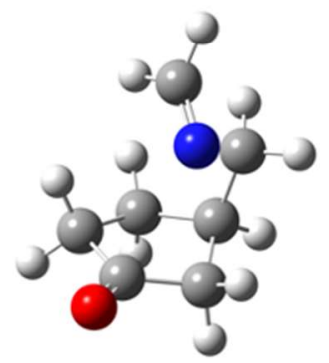

Shoulder

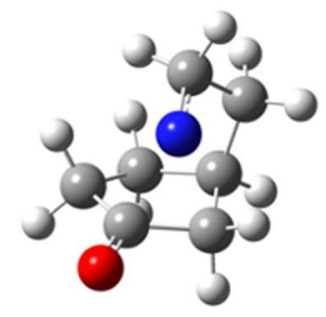

TS

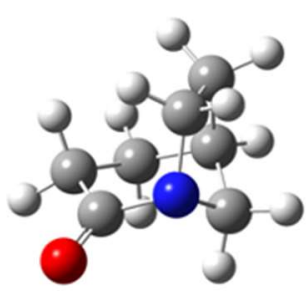

Product

Figure S2-1. Change in energy relative to the product (black dots) and sum of squared singular values of $\mathbf{U}_{\mathrm{VO}}^{(1)}$ (red dots) along the IRC of Schmidt-Aubé reaction. ${ }^{1}$ The IRC was calculated for the singlet ground state at B3LYP/6-311++G(d,p) level. The structures at the reactant, shoulder, TS, and product are also shown below the graph. The sum of squared singular values of $\mathbf{U}_{\mathrm{VO}}^{(1)}$ has two peaks: the peak at $-7.95 \mathrm{amu}^{1 / 2}$ bohr (shoulder) and the peak near the TS $\left(0.00 \mathrm{amu}^{1 / 2} \mathrm{bohr}\right)$. Though there is no intermediate, two asynchronous processes occur in this reaction. 


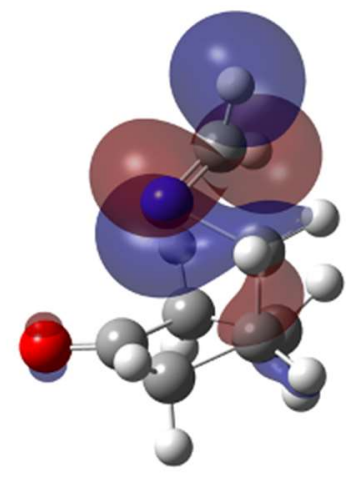

$\phi_{1}$

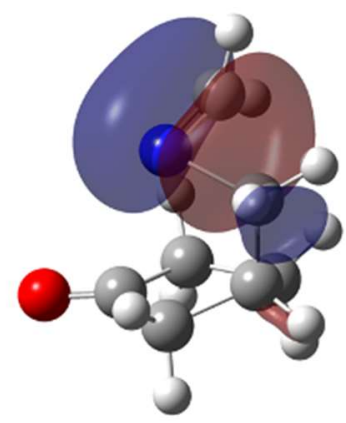

$\phi_{2}$

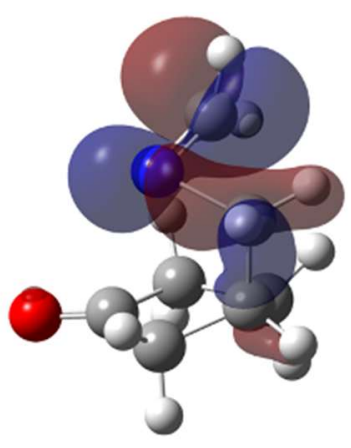

$\phi_{1}^{\prime}$

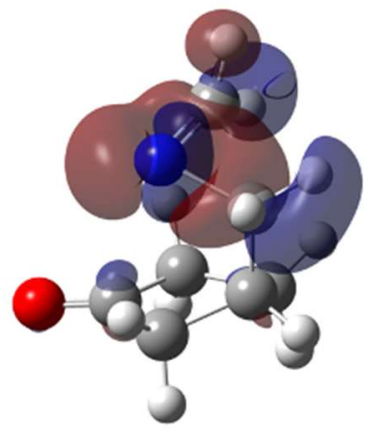

$\phi_{2}^{\prime}$

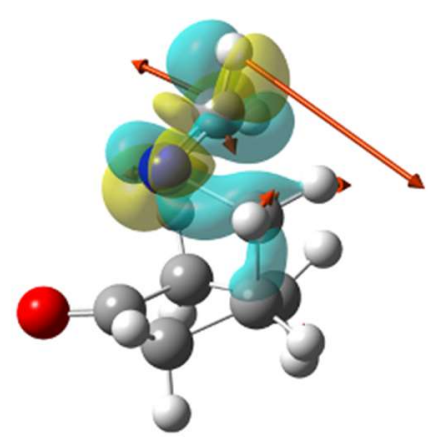

$\phi_{1}^{\prime} \phi_{1}$

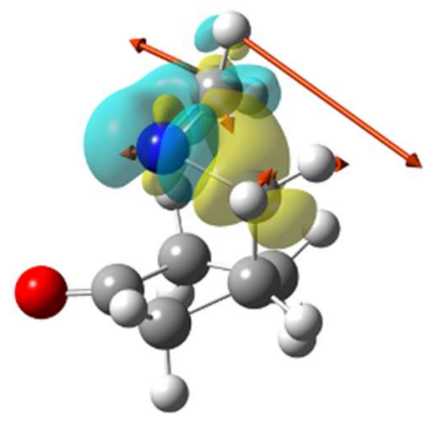

$\phi_{2}^{\prime} \phi_{2}$

Figure S2-2. NRO pairs with the largest and second largest singular values and their products at the shoulder in Fig. S2-1 of Schmidt-Aubé reaction. The products of the NRO pairs are shown together with the tangent vector of the IRC. The singular value and contribution ratio of $\phi_{1}$ and $\phi_{1}^{\prime}$ are 0.571 $\mathrm{amu}^{-1 / 2} \mathrm{bohr}^{-1}$ and $94.0 \%$, respectively, while the singular value and contribution ratio of $\phi_{2}$ and $\phi_{2}^{\prime}$ are $0.114 \mathrm{amu}^{-1 / 2} \mathrm{bohr}^{-1}$ and $3.8 \%$, respectively. Thus, these two sets of NROs cover $97.8 \%$ of the virtual-occupied mixing. Around the shoulder, 1,2-alkyl shift (C-N bond cleavage and C-C bond formation) proceeds. The NRO products, $\phi_{1} \phi_{1}^{\prime}$ and $\phi_{2} \phi_{2}^{\prime}$, show the density decrease in the C-N $\sigma$ bond region and increase in the $\mathrm{C}-\mathrm{C} \sigma$ bond region, respectively. Thus, the mixture of $\phi_{1}$ and $\phi_{1}^{\prime}$ contributes to the $\mathrm{C}-\mathrm{N}$ bond cleavage and the mixture of $\phi_{2}$ and $\phi_{2}^{\prime}$ contributes to the $\mathrm{C}-\mathrm{C}$ bond formation. 

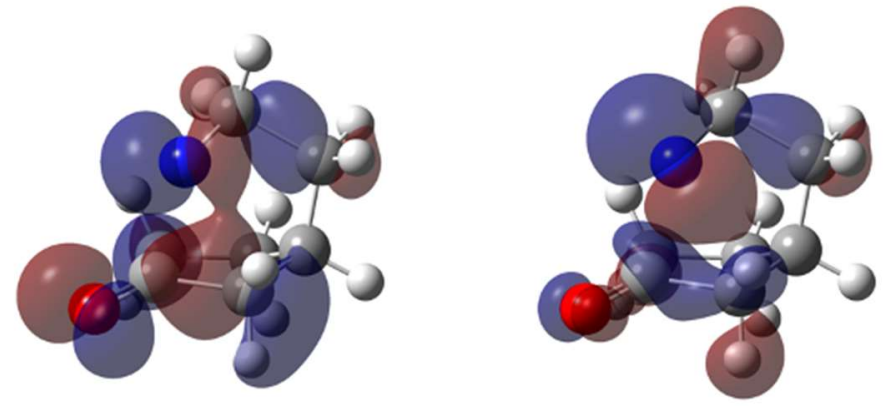

$$
\phi_{1}
$$

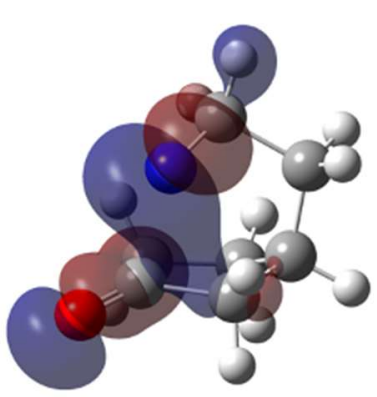

$\phi_{2}$

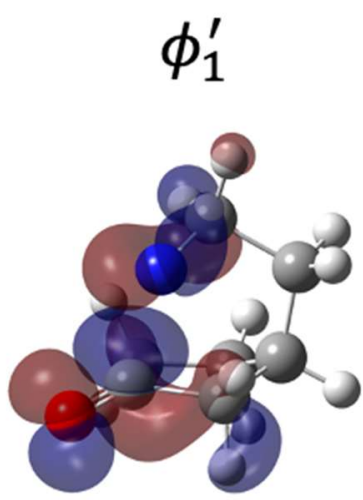

$\phi_{2}^{\prime}$

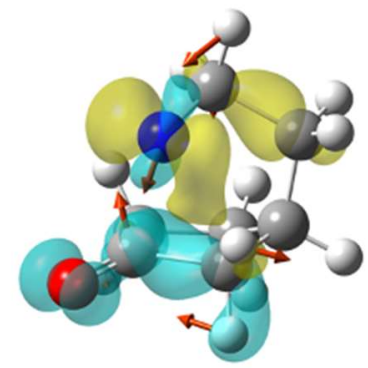

$\phi_{1}^{\prime} \phi_{1}$

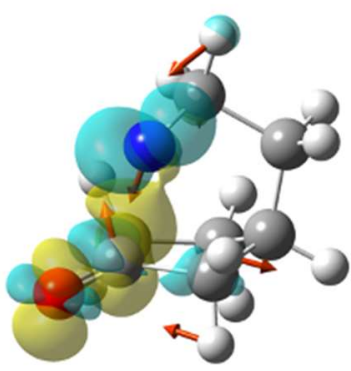

$\phi_{2}^{\prime} \phi_{2}$

Figure S2-3. NRO pairs with the largest and second largest singular values and their products at the TS in Fig. S2-1 of Schmidt-Aubé reaction. The products of the NRO pairs are shown together with the transition vectors with an imaginary frequency. The singular value and contribution ratio of $\phi_{1}$ and $\phi_{1}^{\prime}$ are $0.241 \mathrm{amu}^{-1 / 2} \mathrm{bohr}^{-1}$ and $57.6 \%$, respectively, while the singular value and contribution ratio of $\phi_{2}$ and $\phi_{2}^{\prime}$ are $0.160 \mathrm{amu}^{-1 / 2} \mathrm{bohr}^{-1}$ and $25.6 \%$, respectively. Thus, these two sets of NROs cover $83.2 \%$ of the virtual-occupied mixing. Around the TS, nitrene addition proceeds. The NRO product, $\phi_{1} \phi_{1}^{\prime}$, shows density increase between the nitrogen and one of the $\alpha$-carbon and decrease between the carbonyl carbon and the $\alpha$-carbon. Thus, electrons between the carbonyl carbon and the $\alpha$-carbon are withdrawn by the nitrogen to form a $\sigma$ bond between nitrogen and the $\alpha$-carbon. On the other hand, $\phi_{2} \phi_{2}^{\prime}$, shows density decrease on the nitrogen and increase between the nitrogen and the carbonyl carbon. Thus, electrons on the nitrogen are withdrawn by the carbonyl group to form a $\sigma$ bond between nitrogen and the carbonyl carbon. 


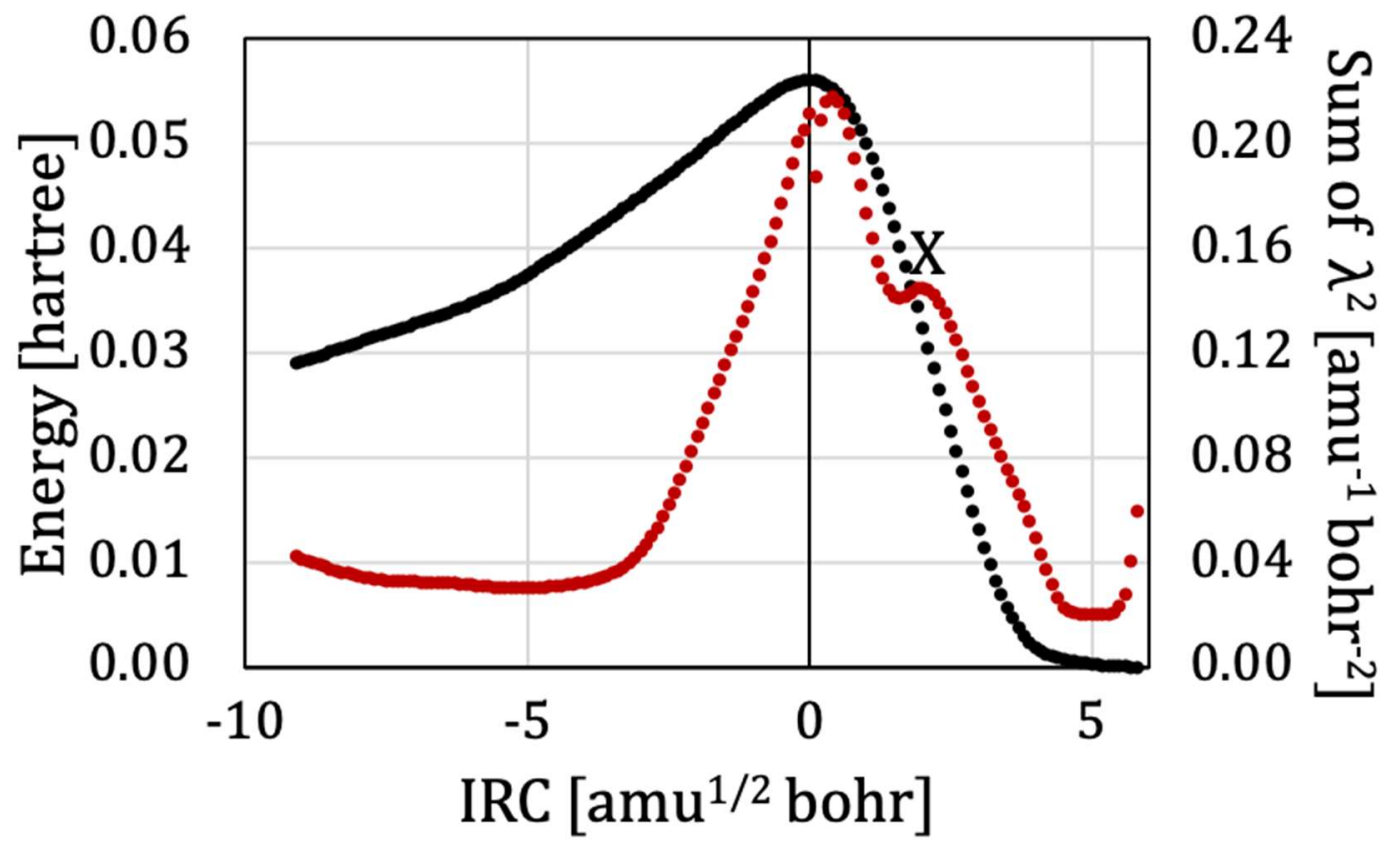

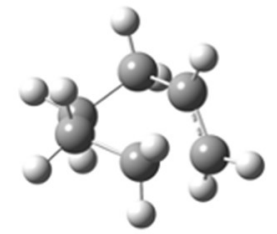

Reactant

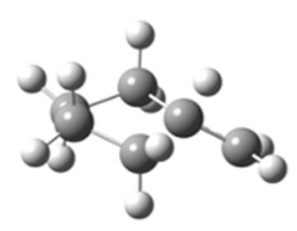

TS

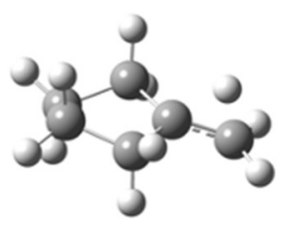

$\mathrm{X}$

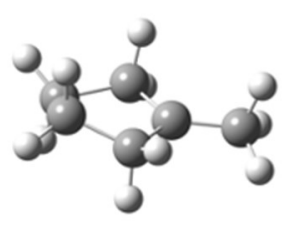

Product

Figure S3-1. Change in energy relative to the product (black dots) and sum of squared singular values of $\mathbf{U}_{\mathrm{VO}}^{(1)}$ (red dots) along the IRC of carbocation rearrangement. The IRC was calculated for the singlet ground state at B3LYP/6-311G(d,p) level with a net charge +1 . The structures at the reactant, TS, X, and product are also shown below the graph. The sum of squared singular values of $\mathbf{U}_{\mathrm{VO}}^{(1)}$ has two peaks: the peak near the TS $\left(0.00 \mathrm{amu}^{1 / 2}\right.$ bohr) and the peak at $2.00 \mathrm{amu}^{1 / 2}$ bohr (shown by X). Though the point $\mathrm{X}$ is not a shoulder of total energy profile, $\mathrm{X}$ is a local maximum of the sum of squared singular values of $\mathbf{U}_{\mathrm{VO}}^{(1)}$. Though there is no intermediate through the reaction, two asynchronous processes take place in this reaction. 

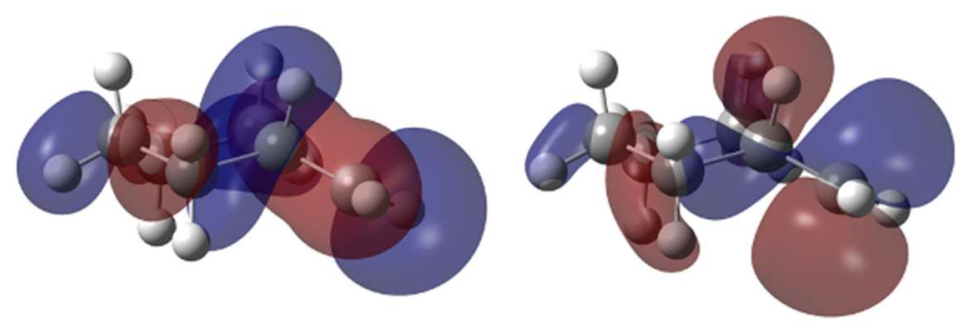

$$
\phi_{1}
$$

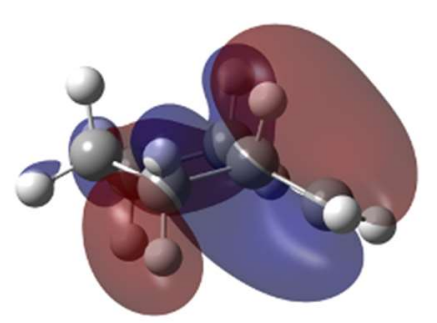

$\phi_{2}$

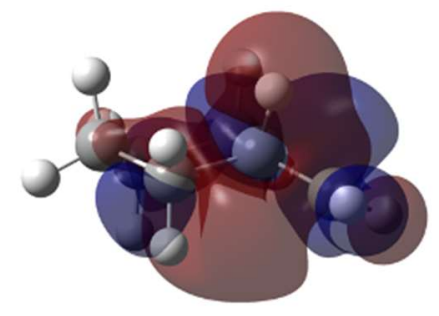

$\phi_{2}^{\prime}$

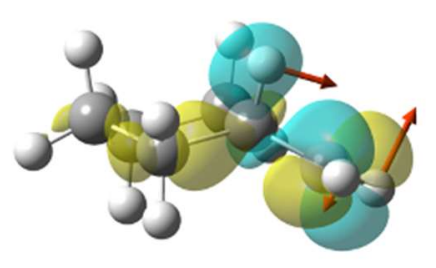

$\phi_{1}^{\prime} \phi_{1}$

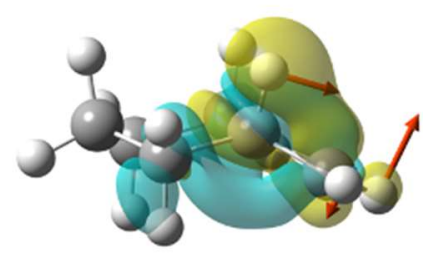

$\phi_{2}^{\prime} \phi_{2}$

Figure S3-2. NRO pairs with the largest and second largest singular values and their products at the TS in Fig. S3-1 of carbocation rearrangement. The products of the NRO pairs are shown together with the transition vectors with an imaginary frequency. The singular value and contribution ratio of $\phi_{1}$ and $\phi_{1}^{\prime}$ are $0.436 \mathrm{amu}^{-1 / 2} \mathrm{bohr}^{-1}$ and $90.0 \%$, respectively, while the singular value and contribution ratio of $\phi_{2}$ and $\phi_{2}^{\prime}$ are $0.106 \mathrm{amu}^{-1 / 2} \mathrm{bohr}^{-1}$ and $5.4 \%$, respectively. Thus, these two sets of NROs cover $95.4 \%$ of the virtual-occupied mixing. The NRO products, $\phi_{1} \phi_{1}^{\prime}$ and $\phi_{2} \phi_{2}^{\prime}$, show the density decrease on the hydride donating carbon and increase on the hydride accepting carbon, respectively. Thus, the mixture of $\phi_{1}$ and $\phi_{1}^{\prime}$ contributes to the $\mathrm{C}-\mathrm{H}$ bond cleavage and the mixture of $\phi_{2}$ and $\phi_{2}^{\prime}$ contributes to the $\mathrm{C}-\mathrm{H}$ bond formation. 


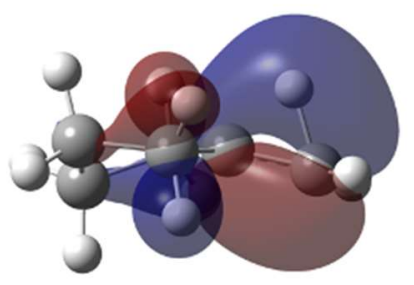

$\phi_{1}$

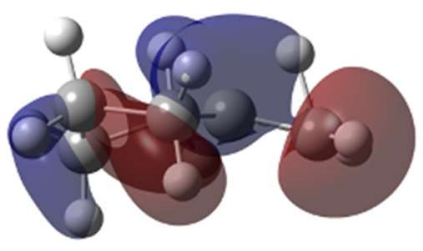

$$
\phi_{2}
$$

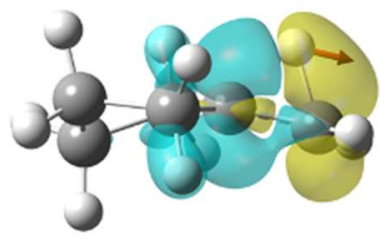

$\phi_{1}^{\prime} \phi_{1}$
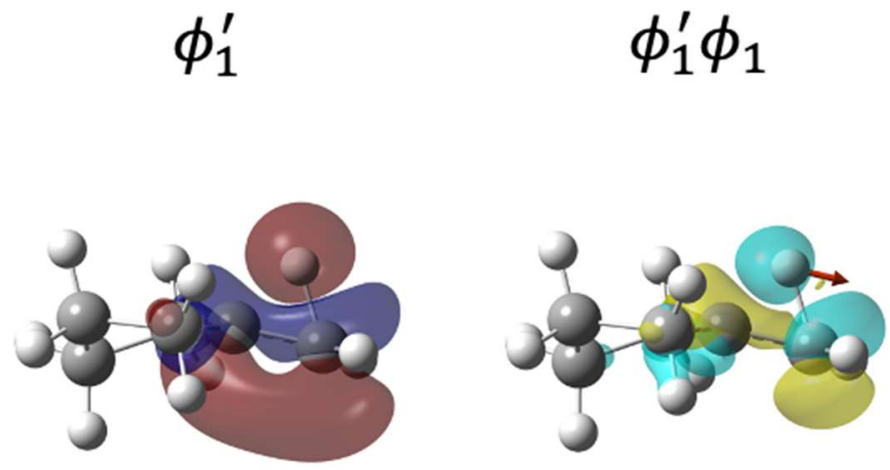

Figure S3-3. NRO pairs with the largest and second largest singular values and their products at the point X in Fig. S3-1 of carbocation rearrangement. The products of the NRO pairs are shown together with the tangent vector of the IRC. The singular value and contribution ratio of $\phi_{1}$ and $\phi_{1}^{\prime}$ are 0.336 $\mathrm{amu}^{-1 / 2} \mathrm{bohr}^{-1}$ and $78.1 \%$, respectively, while the singular value and contribution ratio of $\phi_{2}$ and $\phi_{2}^{\prime}$ are $0.143 \mathrm{amu}^{-1 / 2} \mathrm{bohr}^{-1}$ and $14.2 \%$, respectively. Thus, these two sets of NROs cover $92.3 \%$ of the virtual-occupied mixing. The NRO product, $\phi_{1} \phi_{1}^{\prime}$, shows the density decrease on the hydride donating carbon and increase on the hydride accepting carbon, respectively. Contrary, $\phi_{2} \phi_{2}^{\prime}$, shows the density increase on the hydride donating carbon and decrease on the hydride accepting carbon, respectively. Thus, the mixture of $\phi_{1}$ and $\phi_{1}^{\prime}$ contributes to the 1,2-hydride shift while the mixture of $\phi_{2}$ and $\phi_{2}^{\prime}$ may be considered as stabilization of carbocation center by hyperconjugation. 


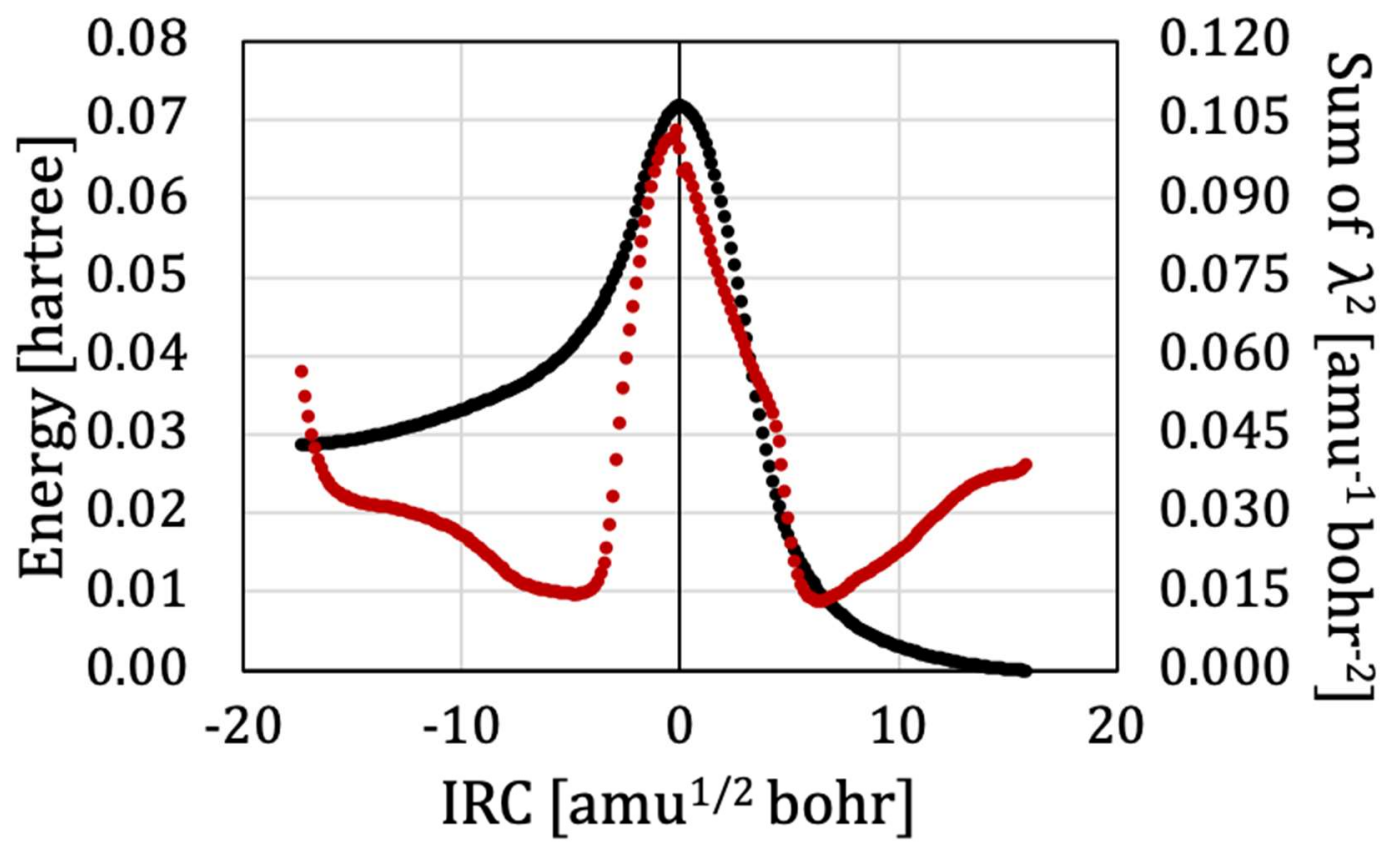

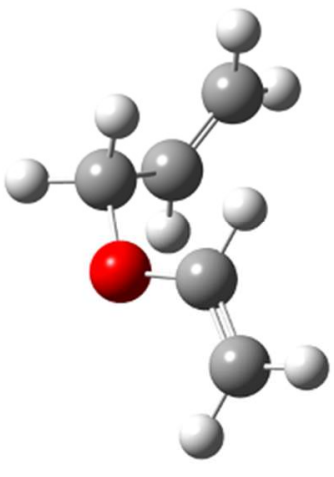

Reactant

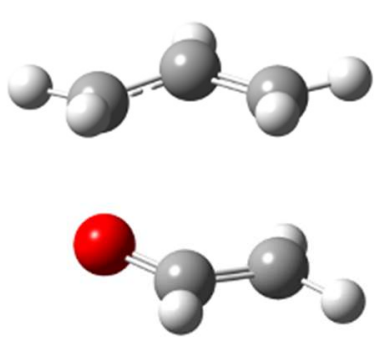

TS

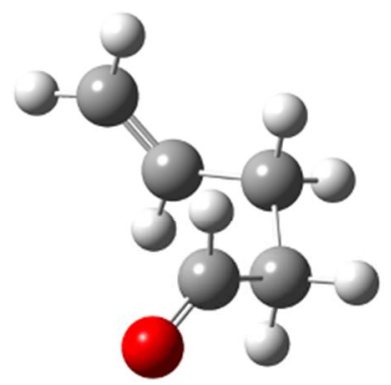

Product

Figure S4-1. Change in energy relative to the product (black dots) and sum of squared singular values of $\mathbf{U}_{\mathrm{VO}}^{(1)}$ (red dots) along the IRC of the Claisen rearrangement. The IRC was calculated for the singlet ground state at $\mathrm{B} 3 \mathrm{LYP} / 6-311+\mathrm{G}(\mathrm{d}, \mathrm{p})$ level. The structures at the reactant, TS, and product are also shown below the graph. The sum of squared singular values of $\mathbf{U}_{\mathrm{VO}}^{(1)}$ have one peak near the TS $(0.00$ $\mathrm{amu}{ }^{1 / 2}$ bohr). Though the sum of squared singular values of $\mathbf{U}_{\mathrm{VO}}^{(1)}$ is also large in reactant and product regions, NRO mixture in these regions are related to relatively unimportant conformation changes. 

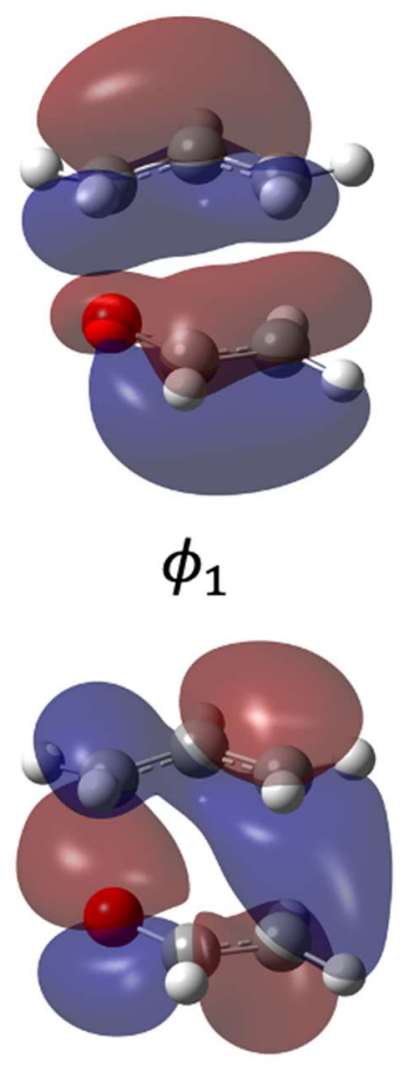

$\phi_{2}$
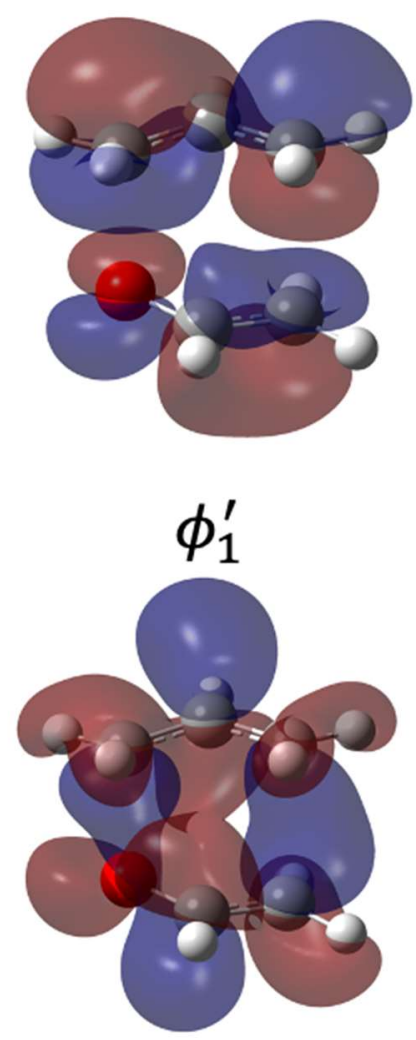

$\phi_{2}^{\prime}$

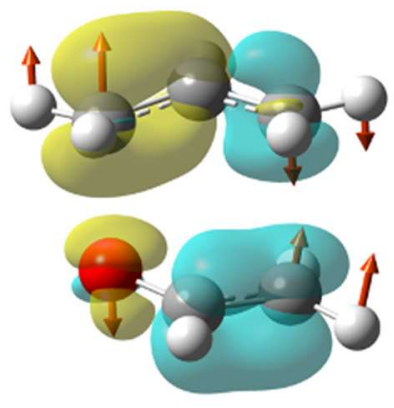

$\phi_{1}^{\prime} \phi_{1}$

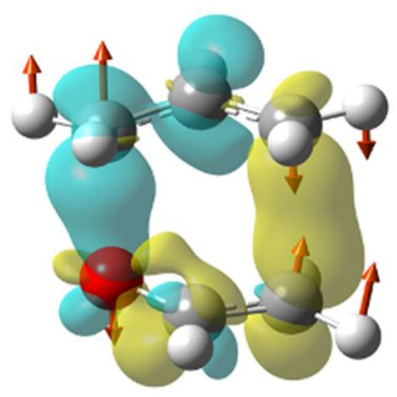

$\phi_{2}^{\prime} \phi_{2}$

Figure S4-2. NRO pairs with the largest and second largest singular values and their products at the TS in Fig. S4-1 of Claisen rearrangement (same to Figure A4). The products of the NRO pairs are shown together with the transition vectors with an imaginary frequency. The singular value and contribution ratio of $\phi_{1}$ and $\phi_{1}^{\prime}$ are $0.228 \mathrm{amu}^{-1 / 2} \mathrm{bohr}^{-1}$ and $52.0 \%$, respectively, while the singular value and contribution ratio of $\phi_{2}$ and $\phi_{2}^{\prime}$ are $0.187 \mathrm{amu}^{-1 / 2} \mathrm{bohr}^{-1}$ and $35.1 \%$, respectively. Thus, these two sets of NROs cover $87.1 \%$ of the virtual-occupied mixing. The NRO product, $\phi_{1} \phi_{1}^{\prime}$, shows density increase and decrease in $\mathrm{C}-\mathrm{C} \pi$ bond regions of the $\mathrm{C}_{3} \mathrm{H}_{5}$ and $\mathrm{OC}_{2} \mathrm{H}_{3}$ fragments, respectively. On the other hand, $\phi_{2} \phi_{2}^{\prime}$, shows density increase in the region of $\mathrm{C}-\mathrm{C} \sigma$ bond to be formed and decrease in the region of $\mathrm{C}-\mathrm{O} \sigma$ bond to be cleaved. A slight density increase in $\mathrm{C}-\mathrm{O} \pi$ bond region is also observed. 


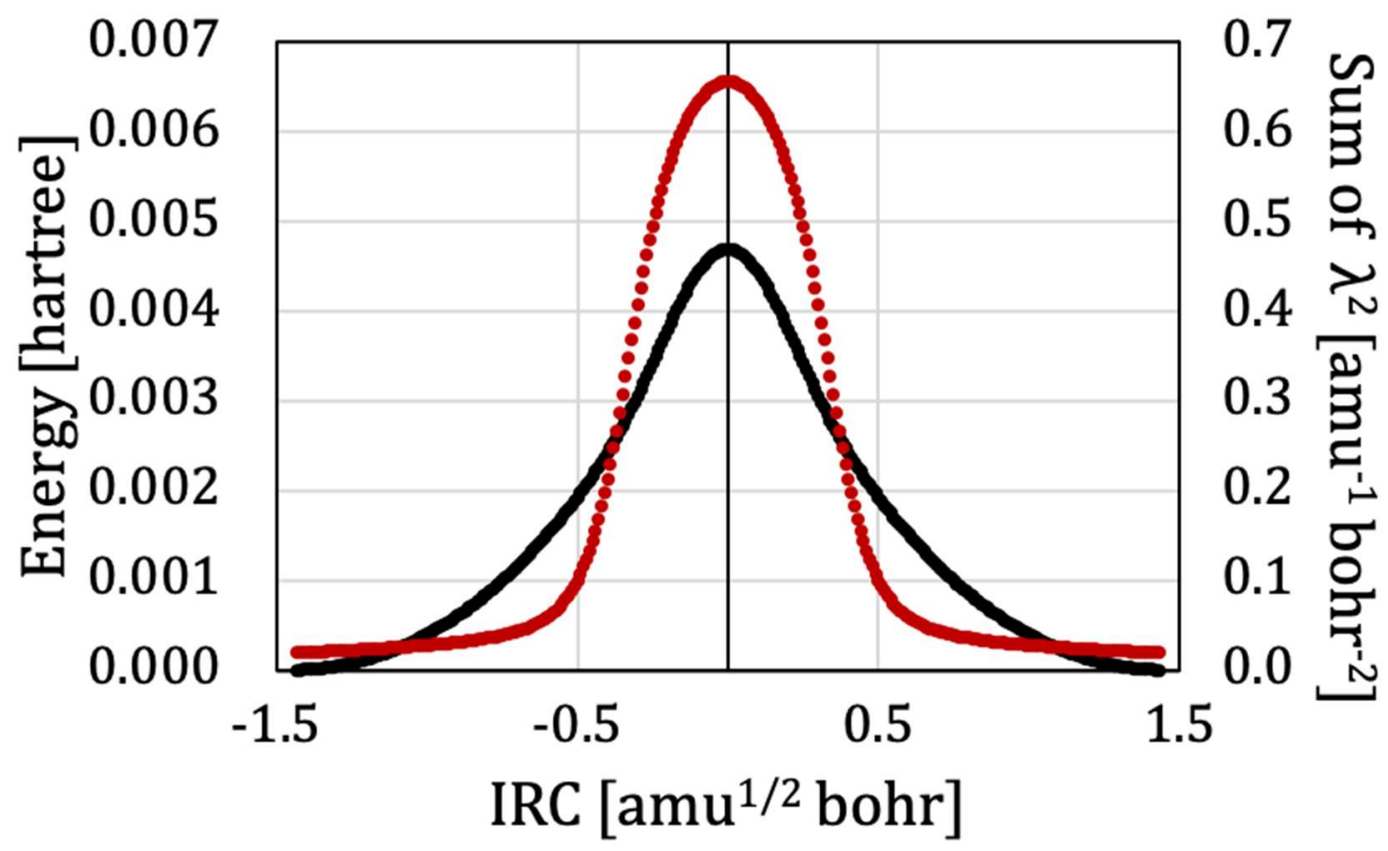

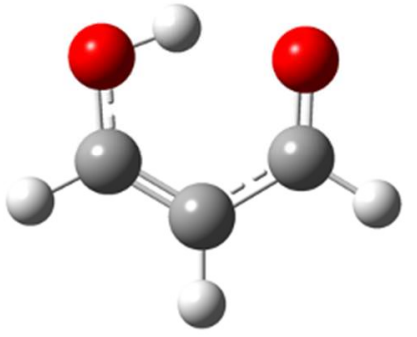

Reactant

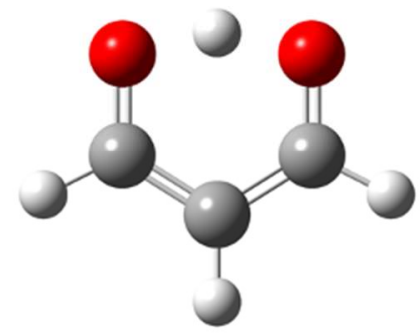

TS

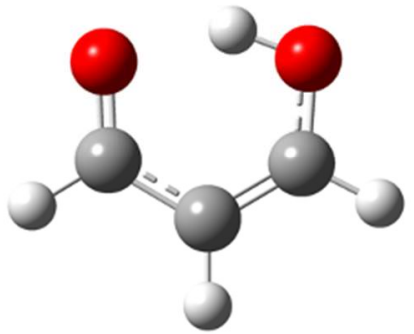

Product

Figure S5-1. Change in energy relative to the product (black dots) and sum of squared singular values of $\mathbf{U}_{\mathrm{VO}}^{(1)}$ (red dots) along the IRC of proton transfer in malonaldehyde. The IRC was calculated for the singlet ground state at LC-BLYP/6-311+G(d,p) level. ${ }^{2}$ The structures at the reactant $\left(\mathrm{C}_{\mathrm{s}}\right)$, TS $\left(\mathrm{C}_{2 \mathrm{v}}\right)$, and product $\left(\mathrm{C}_{\mathrm{s}}\right)$ are also shown below the graph. The sum of squared singular values of $\mathbf{U}_{\mathrm{VO}}^{(1)}$ has one peak near the TS $\left(0.00 \mathrm{amu}^{1 / 2}\right.$ bohr $)$. 

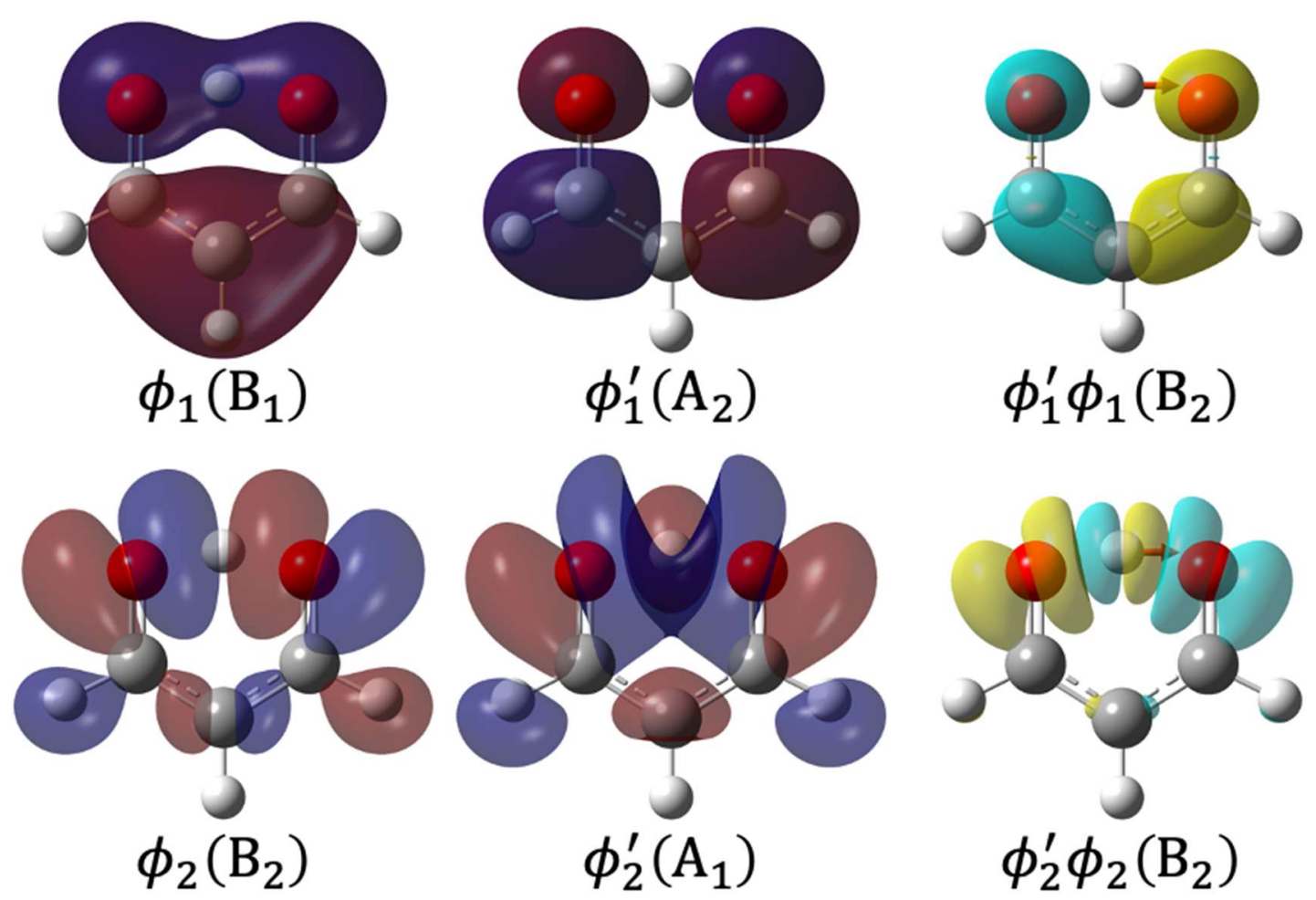

Figure S5-2. NRO pairs with the largest and second largest singular values and their products at the TS $\left(\mathrm{C}_{2 \mathrm{v}}\right)$ in Fig. S5-1 of proton transfer in malonaldehyde. The products of the NRO pairs are shown together with the transition vectors with an imaginary frequency $\left(\mathrm{B}_{2}\right.$ symmetry). Irreducible representations of NROs or NRO products are also shown. The singular value and contribution ratio of $\phi_{1}$ and $\phi_{1}^{\prime}$ are $0.572 \mathrm{amu}^{-1 / 2} \mathrm{bohr}^{-1}$ and $49.9 \%$, respectively, while the singular value and contribution ratio of $\phi_{2}$ and $\phi_{2}^{\prime}$ are $0.523 \mathrm{amu}^{-1 / 2} \mathrm{bohr}^{-1}$ and $41.7 \%$, respectively. Thus, these two sets of NROs cover $91.7 \%$ of the virtual-occupied mixing. The NRO product, $\phi_{1} \phi_{1}^{\prime}$, shows density decrease and increase in the left and right $\mathrm{C}-\mathrm{C} \pi$ bond regions, respectively. This corresponds to the C-C $\pi$ bond shift from left to right. On the other hand, the NRO product, $\phi_{2} \phi_{2}^{\prime}$, shows density increase and decrease on the left and right oxygens, respectively. This corresponds to the cleavage and formation of the right and left $\mathrm{O}-\mathrm{H}$ bonds. 


\section{REFERENCES}

${ }^{1}$ R. B. Campos and D. J. Tantillo, Chem. 5, 227 (2019).

${ }^{2}$ H. Iikura, T. Tsuneda, T. Yanai, and K. Hirao, J. Chem. Phys. 115, 3540 (2001). 